Pacific

Journal of

Mathematics

CLASSIFICATION ET CHANGEMENT DE BASE POUR LES SÉRIES DISCRÈTES DES GROUPES UNITAIRES $p$-ADIQUES

Colette Meglin

Volume $233 \quad$ No. 1

November 2007 


\title{
CLASSIFICATION ET CHANGEMENT DE BASE POUR LES SÉRIES DISCRÈTES DES GROUPES UNITAIRES $p$-ADIQUES
}

\author{
Colette Meglin
}

\begin{abstract}
In this paper we gives the Langlands classification of discrete series using the base change for unitary quasi-split groups and Arthur's idea. This gives a very nice classification of the cuspidal irreducible representations which was not predict by standard conjectures.
\end{abstract}

Il est bien clair pour l'auteur que les méthodes qu'Arthur développe pour les groupes classiques s'appliquent plutôt plus simplement pour les groupes unitaires et cet article n'est donc pas original et il utilise les indications données par Arthur. Son écriture se justifie pour disposer plus rapidement de cas où l'analyse fine que nous faisons des paquets d'Arthur soit utilisable sans conjectures et pour que les hypothèses de [Mœglin 2002] et [Mœglin et Tadić 2002] soient démontrées dans le cas des groupes unitaires. D'autre part, les méthodes que nous employons diffèrent très certainement par endroits des méthodes d'Arthur; ce que nous obtenons et qui n'est pas annoncé par Arthur (même si on pourrait le déduire de ce qui est annoncé) est le fait que l'on caractérise les paquets de Langlands par des propriétés d'irréductibilité de certaines induites, c'est ce que nous avons appelé les blocs de Jordan d'une série discrète.

Ici on suppose que les lemmes fondamentaux ordinaires ou plus exactement le transfert est valide pour les groupes unitaires et leurs groupes endoscopiques et les groupes linéaires tordus comme expliqué ci-dessous et leurs groupes endoscopiques. Les premières hypothèses sont disponibles grâce à [Laumon et Ngo 2004; Waldspurger 1997 ; 2006a]. Les deuxièmes le sont grâce à [Waldspurger 2006b].

Après ce préambule, expliquons ce que nous avons en vue ici : on suppose que $F$ est un corps p-adique et que $E$ est une extension quadratique de $F$ non scindée. On note $\theta$ l'automorphisme extérieur de $\operatorname{GL}(n, E)$ qui à $g \in \operatorname{GL}(n, E)$ associe $J^{t} \bar{g}^{-1} J^{-1}$, où $J$ est la matrice antidiagonale avec à la place $i$ l'élément $(-1)^{i+1}$. On dit qu'une représentation tempérée de $\operatorname{GL}(n, E)$ est $\theta$-discrète si sa classe de conjugaison est invariante sous l'action de $\theta$ et si elle n'est pas une induite propre à partir d'un parabolique $\theta$-stable et d'une représentation $\theta$-invariante

MSC2000: 22E50, 20G05.

Mots-clefs: representations of unitary $p$-adic groups, Langlands classification, cuspidal representations of unitary groups, discrete series of unitary groups. 
de ce parabolique. On considère le groupe produit semi-direct de $\operatorname{GL}(n, E)$ avec le groupe $\{1, \theta\}$ et on note $\tilde{G}_{n}$ la composante de $\theta$ dans ce groupe. Une théorie de l'endoscopie pour $\tilde{G}_{n}$ a été développée par Langlands, Kottwitz et Shelstad; on connait les groupes endoscopiques, ou plutôt les données endoscopiques pour $\tilde{G}_{n}$. Le but de l'article est double : d'abord on montre l'analogue de l'hypothèse d'induction locale [Arthur 2005, p. 244], c'est-à-dire que pour $\pi$ une représentation $\theta$-discrète de $\mathrm{GL}(n, E)$ prolongée en une représentation du produit semi-direct, en une représentation $\tilde{\pi}$, il existe exactement une donnée endoscopique $\langle H\rangle$ de $\tilde{G}_{n}$ tel que $\tilde{\pi}$ soit le changement de base pour un paquet de représentations de $H$ suivant cette donnée endoscopique. Cela permet de définir les paquets stables de séries discrètes pour $U(n, E / F)$, de façon très concrète, un ensemble de séries discrètes $\mathscr{F}$ est un paquet stable, s'il existe une série $\theta$-discrète $\pi$ de $\operatorname{GL}(n, E)$ qui soit le changement de base stable d'une combinaison linéaire convenable d'éléments de $\mathscr{F}$. On caractérise les $\pi$ qui peuvent intervenir à la Langlands par des homomorphismes de $W_{E} \times \operatorname{SL}(2, \mathbb{C})$ dans le groupe $\mathrm{GL}(n, \mathbb{C})$. Puis on montre que l'ensemble des séries discrètes de $U(n, E / F)$ est la réunion disjointe des paquets stables et on calcule le nombre d'éléments dans chaque paquet; cela donne une bijection numérique entre les éléments d'un paquet et les caractères d'un groupe (qui peut s'identifier à un centralisateur de $\psi$ dans un groupe convenable). On donne quelques précisions sur cette bijection; en particulier elle vérifie les propriétés de [Mœglin 2002] et [Mœglin et Tadić 2002]. Au passage on donne une classification des représentations cuspidales de $U(n, E / F)$ en termes de paramètres de Langlands. Ceci prouve les hypothèses de base utilisées dans [Mœglin 2002] et [Mœglin et Tadić 2002].

L'endoscopie tordue a été étudiée par Kottwitz et Shelstad [1999] et aussi par Labesse [2004]. On a repris ici les définitions de l'article [Harris et Labesse 2004] qui décrit explicitement les groupes endoscopiques qui interviennent ici. Dans cette référence, les choix sont précisés. Ces choix nous donnent un caractère $\omega$ de $E^{*}$ dont la restriction à $F^{*}$ est le caractère de $F^{*}$ correspondant à l'extension $E$ de $F$. Ainsi le changement de base instable se déduit du changement de base stable par tensorisation par le caractère $\omega$. Plus généralement, on note $D(n)$ l'ensemble des couples ordonnés d'entiers, $n_{1}, n_{2}$ dont l'un peut être nul tels que $n=n_{1}+n_{2}$. Pour $\left(n_{1}, n_{2}\right) \in D(n)$, on note $s_{n_{1}, n_{2}}$ l'élément de $\operatorname{GL}(n, \mathbb{C})$ diagonal dont les $n_{1}$ premières valeurs propres sont 1 et les $n_{2}$ dernières sont -1 . Il définit naturellement un élément du groupe dual de $\operatorname{GL}(n, E) \rtimes\{1, \theta\}$ et donc une donnée endoscopique [Harris et Labesse 2004, 1.4] ; le groupe endoscopique correspondant est $H_{n_{1}, n_{2}}$ le produit des 2 groupes unitaires quasidéployés, $U\left(n_{1}, E / F\right) \times U\left(n_{2}, E / F\right)$. Cela épuise l'ensemble des données endoscopiques pour $\tilde{G}_{n}$ (loc.cit.). Si $n_{2}=0, H_{n, 0}=U(n)$ fournit le changement de base stable tandis que si $n_{1}=0, H_{0, n}=U(n)$ fournit le changement de base instable. Le résultat de cet article sur le changement de base est de montrer qu'à toute représentation 
$\theta$-discrète $\pi$ de $\operatorname{GL}(n, E)$ correspond exactement une donnée endoscopique $n_{1}, n_{2}$ tel qu'il existe $\Pi^{H_{n_{1}, n_{2}}}$ un ensemble de représentations de $H_{n_{1}, n_{2}}$ tel que tr $\tilde{\pi}$ soit un transfert endoscopique d'une distribution de la forme $\sum_{\tau \in \Pi^{H_{1}, n_{2}}} c_{\tau} \operatorname{tr} \tau$ où les $c_{\tau}$ sont des nombres complexes.

Si $F$ était le corps des réels, un résultat analogue a été démontré par Harris et Labesse [2004] en s'appuyant sur [Clozel 1982]; le résultat démontré là-bas se limite au cas où $n_{1} n_{2}=0$.

Revenons au cas où $F$ est un corps p-adique; on utilise l'espace des intégrales orbitales des fonctions cuspidales sur $\tilde{G}_{n}$, noté $I_{\text {cusp }}\left(\tilde{G}_{n}\right)$; cette idée revient à [Arthur 1996]. On définit un espace analogue pour les groupes $H_{n_{1}, n_{2}}$, noté $I_{\text {cusp }}\left(H_{n_{1}, n_{2}}\right)$ et un sous-espace de cet espace formé par les intégrales orbitales stables, $I_{\text {cusp }}^{\text {st }}\left(H_{n_{1}, n_{2}}\right)$. Il faut recopier [Arthur 1996] et [Waldspurger 2007] pour obtenir une décomposition en somme directe :

$$
I_{\text {cusp }}\left(\tilde{G}_{n}\right)=\bigoplus_{\left(n_{1}, n_{2}\right) \in D(n)} I_{\text {cusp }}^{\text {st }}\left(H_{n_{1}, n_{2}}\right) .
$$

L'application de $I_{\text {cusp }}\left(\tilde{G}_{n}\right)$ vers le membre de droite est la somme des transferts (dont on a maintenant l'existence); ici aucun automorphisme extérieur n'apparaît dans la situation. En suivant [Arthur 1996, 3.5] on vérifie que cette application est une isométrie pour un produit scalaire convenablement défini en loc. cit. En suivant nos références et précisément ici [Waldspurger 2007], on sait associer à une représentation tempérée $\theta$-discrète de $\operatorname{GL}(n, E)$ comme ci-dessus un élément de $I_{\text {cusp }}\left(\tilde{G}_{n}\right)$; cet élément, $f_{\tilde{\pi}}$, est obtenu en considérant la projection sur $I_{\text {cusp }}$ d'un pseudo coefficient (qui doit être défini) de l'extension $\tilde{\pi}$. De plus $I_{\text {cusp }}\left(\tilde{G}_{n}\right)$ est linéairement engendré par ces éléments $f_{\tilde{\pi}}$. Le résultat que nous avons en vue, s'exprime exactement par le fait que l'image de $f_{\tilde{\pi}}$ dans le membre de droite de (8-4) est nulle pour tout élément de $D(n)$ sauf précisément l'un d'entre eux.

Le résultat qualitatif précédent se précise de la façon suivante permettant de calculer l'élément $D(n)$ en question : on écrit $\pi$ comme une induite de représentations de Steinberg généralisées :

$$
\pi=\underset{(\rho, a)}{\times} \operatorname{St}(\rho, a)
$$

où $(\rho, a)$ parcourt un ensemble de couples où $\rho$ est une représentation cuspidale irréductible d'un groupe $\operatorname{GL}\left(d_{\rho}, E\right)$, telle que $\rho \simeq{ }^{\theta} \rho$ où $\theta$ est l'analogue du $\theta$ déjà défini pour $n$ remplacé par $d_{\rho}$ et où $a$ est un entier. On dit que $(\rho, a)$ est stable si

$a$ est pair et la fonction $L$ d'Asai-Shahidi associée à $\rho$ a un pôle en $s=0$, ou

$a$ est impair et la fonction $L$ d'Asai-Shahidi associée à $\rho$ n'a pas de pôle en $s=0$.

On entend par fonction $L$ d'Asai-Shahidi la fonction $L$ de [Shahidi 1990] qui contrôle la réductibilité de l'induite de $\rho$ vue comme représentation du parabolique 
de Levi GL $(n, E)$ du groupe déployé $U(2 n, E / F)$. Cette fonction a été étudiée en [Goldberg 1994] et c'est esssentiellement la fonction $L$ d'Asai usuelle. Le "essentiellement" cache un point non trivial : la fonction $L$ d'Asai est associée à une représentation de $W_{F}$ et non $W_{E}$. Il faut donc déjà passer de $\rho$ à une représentation de $W_{F}$ et non pas $W_{E}$. Evidemment ici on n'a pas besoin de cette interprétation, une façon élémentaire de remplacer l'existence d'un pôle est de dire que l'induite de $\rho$ à $U\left(2 d_{\rho}, E / F\right)$ est irréductible (existence d'un pôle) ou non irréductible absence de pôle. C'est un des résultats de Goldberg qui inclut en particulier que soit l'induite de $\rho$ est irréductible soit c'est l'induite de $\omega \otimes \rho$ qui a cette propriété et les deux options sont exclusives l'une de l'autre; voir l'introduction de [Goldberg 1994, (1)]. Dans le cas où $d_{\rho}$ est impair, la situation est plus simple car contrôlée par le caractère central de $\rho$ mais de façon désagréable notre fonction $L$ est en fait une torsion de la fonction $L$ d'Asai usuelle par le caractère quadratique de $W_{F}$ qui correspond à l'extension $E$ de $F$. Pour éviter les confusions on note $L\left(\rho, r_{A}^{\prime}, s\right)$ cette fonction $L$ indépendamment de la parité de $d_{\rho}$.

Avec les notations ci-dessus, on note $\Phi_{\pi}^{\text {st }}$ l'ensemble des couples $(\rho, a)$ apparaissant dans (0-2) tels que $(\rho, a)$ soit stable. Alors $f_{\tilde{\pi}}$ a une projection nulle dans $I_{\text {cusp }}^{\text {st }}\left(H_{n_{1}, n_{2}}\right)$ sauf exactement si $n_{1}=\sum_{(\rho, a) \in \mathcal{G}_{\pi}^{\text {st }}} a d_{\rho}$.

On sait donc maintenant ce qu'est une représentation elliptique stable de $\tilde{G}_{n}$; elle est associée à un morphisme $\psi$ de $W_{E} \times \operatorname{SL}(2, \mathbb{C})$ dans $\operatorname{GL}(n, \mathbb{C}), \theta$-invariant, sans multiplicité et qui se décompose en somme de représentations irréductibles correspondant à des séries discrètes $\operatorname{St}(\rho, a)$ (voir ci-dessus) telles que $(\rho, a)$ soit stable. On note $\Pi(\psi)$ le paquet de séries discrètes de $U(n, E / F)$ associées à $\psi$. On montre que $|\Pi(\psi)|=2^{\ell(\psi)-1}$, où $\ell(\psi)$ est la longueur de $\psi$ vu comme représentation de $W_{E} \times \operatorname{SL}(2, \mathbb{C})$. On montre aussi que $\Pi(\psi)$ ne contient de représentations cuspidales que si $\psi$ est sans trou; on peut exprimer cette condition de la façon suivante. Soit $a$ un entier, on note $\psi[a]$ la composante isotypique de la représentation $\psi$ pour la représentation irréductible de $\operatorname{SL}(2, \mathbb{C})$ de dimension $a$. Pour tout $a$, $\psi[a]$ est naturellement une représentation de $W_{E}$. On dit que $\psi$ est sans trou si pour tout $a>2, \psi[a]$ est une sous-représentation de $\psi[a-2]$ en tant que représentation de $W_{E}$. En termes concrets, cela dit que si $\operatorname{St}(\rho, a)$ est une des composantes de $\pi(\psi)$, avec $a>2$, alors $\operatorname{St}(\rho, a-2)$ en est aussi une. Pour finir, on associe à tout élément de $\Pi(\psi)$ un caractère d'un sous-groupe de $\left(\operatorname{Cent}_{\mathrm{GL}(n, \mathbb{C})} \psi\right)^{\theta}$ (on reprend les idées de [Mœglin 2002]); ce sous-groupe et ce caractère sont canoniquement définis et le caractère reflète les propriétés des modules de Jacquet des éléments de $\Pi(\psi)$. En particulier un tel sous-groupe admet au plus un caractère alterné (on ne donne pas la définition dans cette introduction); si $\psi$ est sans trou, l'existence d'un caractère alterné est équivalente à l'existence de représentations cuspidales dans $\Pi(\psi)$ puisque les représentations cuspidales sont alors exactement celles dont 
le caractère associé est ce caractère alternée. Cela permet de calculer exactement le nombre de représentations cuspidales dans un paquet $\Pi(\psi)$. Si $\psi$ n'est pas sans trou, cela se généralise en remplaçant cuspidale par séries discrètes fortement positives [Mœglin 2002].

Les conjectures d'Arthur associent à tout élément de $\Pi(\psi)$ un caractère de tout le groupe; on vérifie que les conditions mises par Arthur assurent que la restriction de ce caractère au sous-groupe que nous avons défini est notre caractère.

\section{Définitions}

1.1. Le groupe GL(n) tordu et ses classes de conjugaison stable. On recopie ici [Waldspurger 2007]. On reprend les notations $n, F, E$ de l'introduction ainsi que $\theta$ l'automorphisme extérieur de $\operatorname{GL}(n, E)$; on appelle $G_{n}^{+}$le produit semi-direct $\mathrm{GL}(n, E) \rtimes\{1, \theta\}$ et comme dans l'introduction, on note $\tilde{G}_{n}$ la composante connexe de $\theta$ dans $G_{n}^{+}$. Soient deux éléments $g, g^{\prime} \in \tilde{G}_{n}$ semi-simples. On suit la définition de Labesse [2004] concernant la conjugaison stable et suivant cette définition $g, g^{\prime}$ sont stablement conjugués si et seulement si il existe $x \in \operatorname{GL}(n, \bar{F}) \rtimes \theta$ tel que $g=x g^{\prime} x^{-1}$ et pour tout élément $\sigma \in \operatorname{Gal}(\bar{F} / F), \sigma\left(x^{-1}\right) x$ est dans le sous-groupe du centralisateur de $g$ engendré par la composante connexe de ce stabilisateur et le commutant de $\tilde{G}$ dans $\operatorname{GL}(n, \bar{F})$. Supposons maintenant que $g$ est fortement régulier, c'est-à-dire que son centralisateur est commutatif et la composante neutre de ce centralisateur est un tore. On note $\tilde{G}_{n \text {,reg }}$ l'ensemble des éléments fortement réguliers de $\tilde{G}_{n}$.

On dit que $g$ est elliptique si $g Z_{\mathrm{GL}(n, E)}(g)^{0}$ n'est inclus dans aucun sous-groupe de Levi de $\tilde{G}_{n}$; il faut donc préciser ce que l'on entend par sous-groupe de Levi de $\tilde{G}_{n}$; on considère les sous-groupes de Levi $\theta$-stables, $M$, de $\operatorname{GL}(n, E)$, standard et on définit aisément $\tilde{M}$; ces groupes $\tilde{M}$ forment l'ensemble des sous-groupes de Levi standard de $\tilde{G}_{n}$. Ce sont ces sous-groupes de Levi qui interviennet dans ce travail mais pour définir la notion d'elliptique il faut considérer tous les sousgroupes conjugués de ces sous-groupes de Levi standard. On note $\tilde{G}_{n, \text { ell }}$ l'ensemble des éléments fortement réguliers et elliptiques de $\tilde{G}$. Pour toute fonction $f$ localement constante à support compact sur $\tilde{G}$, on sait définir l'intégrale orbitale $J^{\mathrm{GL}(n, E)}(g, f)$ pour tout $g \in \tilde{G}_{\text {reg. }}$. On dit que $f$ est une fonction cuspidale si l'intégrale orbitale $J^{\mathrm{GL}(n, E)}(g, f)=0$ pour tout $g \in \tilde{G}_{n \text {,reg }}-\tilde{G}_{n \text {,ell }}$. On note $C_{\text {cusp }}\left(\tilde{G}_{n}\right)$ l'ensemble des fonctions cuspidales. On note $I_{\text {cusp }}(\tilde{G})$ l'ensemble des applications

$$
I(f): \quad g \in \tilde{G}_{n, \mathrm{ell}} \mapsto J^{\mathrm{GL}(n, E)}(g, f)
$$

pour $f$ parcourant $I_{\text {cusp }}(\tilde{G})$. C'est un espace vectoriel.

1.2. Pseudo-coefficients. Pour pouvoir utiliser [Waldspurger 2007] qui étend la théorie de Schneider et Stuhler au groupe non connexe, il faut juste changer une 
définition, celle de $Z_{2}$ avec la notation de loc. cit. Dans la théorie de [Schneider et Stuhler 1997] il faut que le centre du groupe soit compact et l'idée de Waldspurger est donc de ne considérer que des éléments invariants sous l'action du groupe engendré par l'élément $z_{2}:=z(\varpi) \theta(z(\varpi))^{-1}$ où ici $\varpi$ est une uniformisante de $E$ et $z(\varpi)$ est la matrice diagonale de $\operatorname{GL}(n, E)$ de coefficients diagonaux tous égaux à $\varpi$. La remarque sous-jacente est que l'on s'intéresse aux représentations $\theta$-discrètes irréductibles de $\operatorname{GL}(n, E)$; une telle représentation a donc un caractère central invariant par $\theta$ et $z_{2}$ y agit donc trivialement. On note donc ici $Z_{2}$ le sousgroupe de $\operatorname{GL}(n, E)$ engendré par $z_{2} ; Z_{2}$ est invariant sous l'action de $\theta$ et agit par multiplication à droite sur $\tilde{G}$. L'autre remarque, provenant de [Waldspurger 2007, fin de II.2], est que la multiplication à droite par $z_{2}^{-1}$ n'est autre que la conjugaison sous $z(\varpi)$. Comme on ne travaille qu'avec des objets invariants par conjugaison sous $\mathrm{GL}(n, E)$, ces objets sont naturellement invariants par multiplication à droite par le groupe $Z_{2}$. On peut alors recopier le corollaire [Waldspurger 2007, II.2] en se limitant aux représentations irréductibles. Dans l'énoncé ci-dessous $\Delta$ est le déterminant de Weyl usuel.

Théorème. Soit $\tilde{\pi}$ une représentation irréductible de $G_{n}^{+}$dont la restriction à $\operatorname{GL}(n, E)$ reste irréductible. Alors il existe une fonction $f_{\tilde{\pi}} \in C_{\text {cusp }}(\tilde{G})$ tel que pour tout élément $g \in \tilde{G}_{n, \mathrm{ell}}$ :

$$
\operatorname{tr} \tilde{\pi}(g)=\Delta(g)^{-1 / 2} J^{\mathrm{GL}(n, E)}\left(g, f_{\tilde{\pi}}\right) .
$$

1.3. Représentations elliptiques. On reprend [Waldspurger 2007] qui a été écrit dans un cadre englobant facilement notre cas; c'est ici IV.5 de loc. cit. qui nous intéresse et ce résultat étend des résultats de [Arthur 1993]. Pour toute représentation $\theta$-discrète $\pi$ de $\operatorname{GL}(n, E)$ fixons un prolongement $\tilde{\pi}$ à $G_{n}^{+}$et une fonction cuspidale $f_{\tilde{\pi}}$ satisfaisant au théorème de 1.2. On note $I_{\tilde{\pi}}$ l'image de $f_{\tilde{\pi}}$ dans $I_{\text {cusp }}(\tilde{G})$.

Théorème (Arthur, Waldspurger). L'ensemble des éléments $I_{\tilde{\pi}}$ quand $\pi$ parcourt l'ensemble des représentations $\theta$-discrètes de $\operatorname{GL}(n, E)$ forme une base de $I_{\text {cusp }}(\tilde{G})$.

1.4. Décomposition de $\boldsymbol{I}_{\text {cusp }}(\tilde{\boldsymbol{G}})$. Arthur $[1996,3.5]$ a stabilisé l'espace $I_{\text {cusp }}(G)$ quand $G$ est un groupe réductif connexe quasi-déployé et quand on connaît l'existence du transfert pour tous les groupes endoscopiques elliptiques de $G$. Précisément, le transfert montre que pour tout $f \in I_{\text {cusp }}(G)$ et pour toute donnée endoscopique elliptique $\langle H\rangle$, il existe $f^{\langle H\rangle} \in I_{\text {cusp }}(H)$ tel que $f^{\langle H\rangle}$ soit un transfert de $f$ et soit stable; on peut en plus imposer à $f^{\langle H\rangle}$ d'être invariant sous le groupe d'automorphismes extérieurs de $\langle H\rangle$ provenant de $G$. Ensuite Arthur, pour $G$ connexe quasi-déployé, montre que l'application :

$$
f \in I_{\text {cusp }}(G) \mapsto \bigoplus_{\langle H\rangle} f^{\langle H\rangle}
$$


induit une isométrie de $I_{\text {cusp }}(G)$ sur $\bigoplus_{\langle H\rangle} I_{\text {cusp }}^{\text {st, } \operatorname{Out}_{G}(\langle H\rangle)}(H)$ le produit scalaire sur $I_{\text {cusp }}(G)$ est le produit scalaire ordinaire tandis que le produit scalaire sur le membre de droite est la somme pondérée des produits scalaires ordinaires, les coefficients étant l'analogue local des $i(G, H)$ de Langlands et Kottwitz. La démonstration de [Arthur 1996] s'appuie sur [Waldspurger 1997, 1.2]. Le point clé est de démontrer la surjectivité :

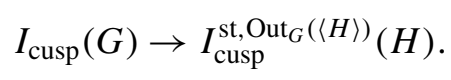

Pour le cas qui nous intéresse ici, Out est trivial ; cela facilite la démonstration. Ceci a été repris dans le cadre non connexe par [Waldspurger 2007, V] mais où seul le groupe endoscopique principal (celui qui contrôle la stabilité) est traité. C'est cette démonstration dont on va vérifier qu'elle se généralise. Comme l'a remarqué Arthur le point de départ est le transfert au niveau des algèbres de Lie c'est à dire la formule [Waldspurger 1997, 1.2], maintenant valable dans le cas de $\tilde{G}$ et $\langle H\rangle$ une donnée endoscopique générale grâce à [Waldspurger 2006b].

Il y a deux points dans cette démonstration. Le premier point consiste à définir $I_{\text {cusp }}^{\langle H\rangle \text {-st }}(\tilde{G})$ comme sous-espace de $I_{\text {cusp }}(\tilde{G})$; ce sont l'ensemble des fonctions sur les intégrales orbitales elliptiques qui à l'intérieur d'une classe de conjugaison stable se transforment via le facteur de transfert relatif à la donnée $\langle H\rangle$. Et il faut démontrer la décomposition (en tenant compte du fait que Out est trivial) :

$$
I_{\text {cusp }}(\tilde{G})=\bigoplus_{\langle H\rangle} I_{\text {cusp }}^{\langle H\rangle \text {-st }}(\tilde{G}) .
$$

Le deuxième point consiste à montrer que le transfert identifie $I_{\text {cusp }}^{\langle H\rangle \text {-st }}(\tilde{G})$ et $I_{\text {cusp }}^{\text {st }}(H)$. Pour ce deuxième point on peut reprendre la démonstration formelle d'Arthur [1996] puisque Out est trivial ou celle de [Waldspurger 2007, VI.1] en y remplaçant "stable" par " $\langle H\rangle$-stable".

Pour le premier point, on fixe $\langle H\rangle$ une donnée endoscopique elliptique de $\tilde{G}$ et $I$ un élément $\langle H\rangle$-stable de $I_{\text {cusp }}(\tilde{G})$, on doit montrer qu'il existe $f \in C_{\text {cusp }}(\tilde{G})$ tel que $I$ soit la fonction associée aux intégrales orbitales de $f$.

Un argument de partition de l'unité ramène à faire cette démonstration localement près des points semi-simples elliptiques.

Fixons un élément semi-simple elliptique de $\tilde{G}$ ou plus exactement sa classe de conjugaison stable. On a donc un ensemble fini de classes de conjugaison à l'intérieur de cette classe de conjugaison stable et il faut contruire la fonction cherchée au voisinage de chacun de ces points de façon compatible à la conjugaison stable. On est donc amené à travailler au voisinage de 0 dans l'algèbre de Lie du centralisateur de chacun de ses points et la première difficulté est que les centralisateurs ne sont pas isomorphes mais sont des formes intérieures l'un de l'autre. La remarque qui permet de travailler a été faite en [Waldspurger 2007, V.3]; le torseur qui ramène à la forme quasidéployée de ces centralisateurs est 
compatible à la conjugaison stable. Cette remarque est reprise dans le cadre général en [Waldspurger 2006b, paragraphe 3].

On est donc ramené à travailler avec la forme quasidéployée de ces centralisateurs mais il faut encore suivre les facteurs de transfert; si la classe stable de vient pas de $\langle H\rangle$, on prend évidemment la fonction 0 et il n'y a rien à faire. Sinon on est ramené à la "définition d'une donnée endoscopique" [Waldspurger 2006b, 3.5]; c'est la généralisation au cas non connexe de l'utilisation de Kottwitz et Shelstad [1999] par Arthur [1996] et s'appuie fortement sur les définitions de la conjugaison stable de Labesse [2004]. Et on a l'égalité des facteurs de transfert nécessaire en [Waldspurger 2006b, 3.9].

A côté de ce qui précède la démonstration au voisinage de l'origine pour des groupes assez généraux est complètement limpide ; c'est [Waldspurger 2007, V.2] où il faut remplacer "stable" par " $\langle H\rangle$-stable".

1.5. Identité de caractères. Le résultat principal de [Arthur 1996] consiste à montrer qu'une identité de caractères obtenue sur les points elliptiques pour des représentations elliptiques se prolonge en une identité de caractères. Ceci reste vrai au moins pour "stable" dans la situation présente d'après [Waldspurger 2007, VI.3].

Proposition. Soit $\tilde{\Pi}$ une représentation virtuelle combinaison linéaire de représentations elliptiques de $G_{n}^{+}$. Soit $\langle H\rangle$ une donnée endoscopique elliptique de $\tilde{G}$ et $\Pi_{\langle H\rangle}$ une représentation virtuelle de $\langle H\rangle$ combinaison linéaire de représentations elliptiques. Supposons que $\operatorname{tr} \tilde{\Pi}$ soit un transfert de $\operatorname{tr} \Pi_{\langle H\rangle}$ sur les éléments elliptiques réguliers. Alors $\operatorname{tr} \tilde{\Pi}$ est un transfert de $\operatorname{tr} \Pi_{\langle H\rangle}$.

On reprend [Waldspurger 2007, V.5] : on globalise les groupes, pour cela il n'y a pas de problème; en suivant le même article, on note $u$ la place qui nous intéresse. On fixe deux places, $v_{1}, v_{2}$ du corps global différentes de $u$ et en ces places on ne regardera que des fonctions cuspidales. On fixe encore une troisième place finie, $v_{3}$ différente des places déjà utilisées; en cette place on fixe une fonction cuspidale $\langle H\rangle$-stable (cf. la preuve de 1.4). On peut utiliser la formule des traces simples d'Arthur grâce à un résultat de Mezo [2004] comme expliqué en loc.cit.

On est alors dans une situation étudiée en particulier par Labesse et on sait stabiliser la partie elliptique du côté géométrique de la formule des traces grâce à [Labesse 2004]. Avec les fonctions test satisfaisant aux 3 propriétés ci-dessus, seule la donnée endoscopique $\langle H\rangle$ intervient. Soient donc $f_{\tilde{G}}$ et $f_{H}$ des fonctions test sur $\tilde{G}$ et $H$ respectivement cuspidales aux 2 places fixées ci-dessus et à la troisième place $f_{\tilde{G}}$ cuspidale et $\langle H\rangle$-stable tandis que $f_{H}$ est stable; avec la formule des traces simplifiées on montre alors que $f_{H}$ est un transfert de $f_{\tilde{G}}$ si et seulement si la trace de $f_{\tilde{G}}$ est égale à la trace pour $f_{H}$; c'est la démonstration faite dans loc. cite. En suivant toujours Waldspurger [2007, IV.5(3)] on sait aussi projeter les composantes à la place $u$ de $f_{\tilde{G}}$ et $f_{H}$ dans l'ensemble des fonctions cuspidales; 
ceci est défini par le fait que sur toute représentation elliptique la trace de la fonction et celle de sa projection coïncident. La démonstration consiste ensuite à montrer (en utilisant deux fois l'équivalence ci-dessus) que si $f_{H}$ est un transfert de $f_{\tilde{G}}$ alors ceci reste vrai en remplaçant les composantes à la place $u$ de ces fonctions test par leur projection cuspidale. En particulier les projections définies sont des fonctions cuspidales, celle sur $\tilde{G}$ étant un transfert de celle sur $H$. On note $f_{\tilde{G}}^{u}$ et $f_{H}^{u}$ les composantes en $u$ de $f_{\tilde{G}}$ et $f_{H}$ et $f_{\text {cusp,tildeG }}^{u}$ et $f_{\text {cusp }, H}^{u}$ les projections. On a donc puisque $\pi$ et $\pi^{H}$ sont des combinaisons linéaires de représentations elliptiques :

$$
\operatorname{tr} \pi\left(f_{\tilde{G}}^{u}\right)=\operatorname{tr} \pi\left(f_{\text {cusp }, \tilde{G}}^{u}\right) ; \quad \operatorname{tr} \pi^{H}\left(f_{H}^{u}\right)=\operatorname{tr} \pi^{H}\left(f_{\text {cusp }, H}^{u}\right) .
$$

On vient de voir que $f_{\text {cusp, } \tilde{G}}^{u}$ est un transfert de $f_{\text {cusp }, H}^{u}$. Et l'égalité $\operatorname{tr} \pi\left(f_{\tilde{G}}^{u}\right)=$ $\operatorname{tr} \pi^{H}\left(f_{H}^{u}\right)$ résulte donc de l'hypothèse.

1.6. Transfert et module de Jacquet. Soit $\tilde{\pi}$ une représentation irréductible elliptique de $\tilde{G}_{n}$. En couplant les sections précédentes, on trouve pour toute donnée endoscopique elliptique $\langle H\rangle$ de $\tilde{G}_{n}$, une représentation virtuelle $\pi^{\langle H\rangle}$ combinaison linéaire de représentations elliptiques de $H$ telle que l'on ait pour tout élément $\gamma \in \tilde{G}_{n, \text { reg }}$

$$
\operatorname{tr} \tilde{\pi}(\gamma)=\sum_{\langle H\rangle} \sum_{\gamma_{H} \in H} \Delta^{\langle H\rangle}\left(\gamma_{H}, \gamma\right) \operatorname{tr} \pi^{\langle H\rangle}\left(\gamma_{H}\right)
$$

Dans la somme de droite les éléments $\gamma_{H}$ parcourt un ensemble de représentants de classes de conjugaison stable. A priori cette égalité n'est vraie que pour les éléments elliptiques mais comme expliqué ci-dessus, elle s'étend à tout élément fortement régulier.

On fixe $M$ un sous-groupe de Levi de $\operatorname{GL}(n, E)$ de la forme

$$
\mathrm{GL}(a, E) \times \mathrm{GL}(n-2 a, E) \times \mathrm{GL}(a, E)
$$

et un parabolique de Levi M ; on prend le parabolique triangulaire par blocs supérieur. On considère un point $\gamma=z \gamma^{\prime}$ où $\gamma^{\prime} \in \tilde{M}$ et où $z$ est un élément de $E^{*}$ identifié au centre du premier GL( $a, E)$. On applique (1-1) à un tel $\gamma$ pour $z$ contractant les sous-groupes du radical unipotent de $P$. On note $\operatorname{res}_{P} \tilde{\pi}$ la restriction de $\pi$ au parabolique $P$ mais que l'on voit comme une représentation de $\tilde{M}$ puisque $\theta$ continue d'agir. Pour $z$ suffisamment dilatant, on obtient en suivant Casselman une égalité :

$$
\operatorname{tr} \tilde{\pi}\left(z \gamma^{\prime}\right)=\delta^{1 / 2}\left(z \gamma^{\prime}\right) \operatorname{tr} \operatorname{res}_{P} \tilde{\pi}\left(z \gamma^{\prime}\right),
$$

où $\delta$ est la fonction module. Il faut faire un calcul analogue pour le membre de droite; on fixe $\langle H\rangle$ une donnée endoscopique. On écrit

$$
H=U\left(n_{1}, E / F\right) \times U\left(n_{2}, E / F\right) .
$$


On se limite aux éléments $\gamma^{\prime}$ qui sont dans $M$ et dont la composante $\operatorname{sur} \operatorname{GL}(a, E) \times$ $1 \times \mathrm{GL}(a, E)$ est elliptique. On note $M_{1}^{H}$ et $M_{2}^{H}$ les sous-groupes de Levi de $H$ isomorphes respectivement à $\operatorname{GL}(a, E) \times U\left(n_{1}-2 a, E / F\right) \times U\left(n_{2}, E / F\right)$ et $U\left(n_{1}, E / F\right) \times \mathrm{GL}(a, E) \times U\left(n_{2}-2 a, E / F\right)$ et $P_{i}^{H}$ les sous-groupes paraboliques "standard" correspondant; $M_{1}^{H}$ n'existe que si $n_{1} \geq 2 a$ et de même pour $M_{2}^{H}$. Soit $\gamma_{H}^{\prime \prime}$ un élément de $H$ tel que $\Delta^{\langle H\rangle}\left(\gamma_{H}^{\prime \prime}, z \gamma^{\prime}\right) \neq 0$. Alors $\gamma_{H}^{\prime \prime}$ est conjugué d'un élément de $M_{1}^{H}$ ou d'un élément de $M_{2}^{H}$, les 2 n'étant pas exclusifs ; on fixe $i=1,2$ tel que $\gamma_{H}^{\prime \prime}$ soit conjugué d'un élément de ce Levi (on considère alors $\gamma_{H}^{\prime \prime}$ comme un élément de ce Levi) et on écrit $\gamma^{\prime \prime}=z \gamma_{H}^{\prime}$ où $z$ est vu comme un élément du facteur $\operatorname{GL}(a, E)$ du Levi dans lequel $\gamma_{H}^{\prime \prime}$ est elliptique. On calcule encore $\operatorname{tr} \pi^{\langle H\rangle}\left(z \gamma_{H}^{\prime}\right)$ à l'aide de la restriction de $\pi^{H}$ au parabolique du Levi fixé. On obtient donc un calcul de $\operatorname{tr} \operatorname{res}_{P} \tilde{\pi}\left(z \gamma^{\prime}\right)$ en fonction de ces restrictions. Les deux termes dépendent de $z$ dans un cône; cette égalité se prolonge à tout $z$ et on peut donc faire $z=1$. Fixons encore $\langle H\rangle$ comme ci-dessus et $\gamma_{H}^{\prime}$, et notons $i=1,2$ l'indice tel que $\gamma_{H}^{\prime}$ soit elliptique dans $M_{i}^{H}$. Il est clair que $M_{i}^{H}$ est naturellement une donnée endoscopique elliptique pour $M$ et le point est de comparer les facteurs de transfert. C'est une situation simple puisque que l'on passe d'un groupe à l'un de ses Levi. Précisons les notations ; on écrit $\gamma^{\prime}=m h m^{\prime} \theta$ avec $m, m^{\prime} \in \mathrm{GL}(a, E)$ et $h \in \mathrm{GL}(n-2 a, E)$ et on suppose que $\gamma_{H}^{\prime}$ est dans $M_{2}^{H}$; on écrit alors $\gamma_{H}^{\prime}=m_{2} h_{H}^{\prime}$ où $m_{2} \in \operatorname{GL}(a, E)$ et $m_{2} \in U\left(n_{1}, E / F\right) \times U\left(n_{2}-2 a, E / F\right)$. On vérifie que si les classes stables de $\gamma_{H}^{\prime}$ et $\gamma^{\prime}$ se correspondent, alors il en est de même de $h \theta$ et $h_{H}^{\prime}$ et $m_{2}$ est dans la même classe de conjugaison que $m \theta\left(m^{\prime}\right)$. Aux fonctions modules près qui disparaissent dans les calculs, on trouve, pour $\omega$ un caractère convenable de $E^{*}$ (cf. introduction)

$$
\Delta^{\langle H\rangle}\left(\gamma_{H}, \gamma\right)=\omega\left(\operatorname{det} m_{2}\right) \Delta^{\left\langle U\left(n_{1} \times U\left(n_{2}-2 a\right)\right\rangle\right.}\left(h_{H}^{\prime}, h \theta\right) .
$$

Si on travaille avec le parabolique $P_{1}^{H}$, il n'y a pas le caractère $\omega$. Le caractère $\omega$ est un caractère de $E^{*}$ qui prolonge le caractère de $F^{*}$ correspondant via le corps de classe à l'extension $E$ de $F$.

Finalement on trouve, avec les notations ci-dessus en y faisant $m^{\prime}=1$ ce qui est loisible et avec $m$ elliptique dans $\operatorname{GL}(a, E)$ :

$$
\text { (1-2) } \operatorname{tr} \operatorname{res}_{P} \tilde{\pi}(m h \theta)=\sum_{\langle H\rangle, i=1,2} \sum_{\gamma_{H}^{\prime}} \Delta^{\left\langle M_{i}^{H}\right\rangle}\left(h_{H}^{\prime}, h \theta\right) \operatorname{tr} \operatorname{res}_{P_{i}^{H}} \pi^{\langle H\rangle}\left(m h_{H}^{\prime}\right) \omega^{i-1}(m) .
$$

On peut en tirer des résultats plus précis en découpant suivant les représentations cuspidales du groupe $\mathrm{GL}(a, E)$ qui interviennent. Il y a des regroupements possibles et donc des simplifications possibles.

On considère $\operatorname{res}_{P} \tilde{\pi}$ comme une représentation de $\mathrm{GL}(a, E) \times \mathrm{GL}(n-2 a, E) \times$ $\mathrm{GL}(a, E)$. Et on décompose cette représentation dans le groupe de Grothendieck 
convenable sous la forme :

$$
\bigoplus_{\sigma, \sigma^{\prime}} \sigma \otimes \pi\left(\sigma, \sigma^{\prime}\right) \otimes \sigma^{\prime}
$$

où $\sigma, \sigma^{\prime}$ parcourt un ensemble de représentants des classes d'isomorphisme de représentations irréductibles de $\operatorname{GL}(a, E)$ et où $\pi\left(\sigma, \sigma^{\prime}\right)$ est une représentation virtuelle de $\operatorname{GL}(n-2 a, E)$. Supposons que $\sigma^{\prime} \simeq{ }^{\theta}(\sigma)$, alors $\pi\left(\sigma,{ }^{\theta}(\sigma)\right)$ a naturellement une action de $\theta$ et est donc une représentation virtuelle de $\tilde{G}_{n-2 a}$, représentation que l'on écrit $\tilde{\pi}\left(\sigma,{ }^{\theta}(\sigma)\right)$. De l'algèbre linéaire simple montre que :

$$
\operatorname{tr} \operatorname{res}_{P} \tilde{\pi}(m h \theta)=\sum_{\sigma} \operatorname{tr} \sigma(m) \operatorname{tr} \tilde{\pi}(\sigma, \theta(\sigma))(h \theta),
$$

où $\sigma$ parcourt un ensemble de représentants des classes d'équivalence de représentations irréductibles de $\operatorname{GL}(a, E)$. De même pour toute représentation virtuelle $\Pi^{\langle H\rangle}$, on écrit, pour $i=1,2$ :

$$
\operatorname{res}_{P_{i}^{H}} \Pi^{H}=\sum_{\sigma} \sigma \otimes \Pi^{H}(\sigma, i) .
$$

On découpe l'égalité (1-2) suivant les représentations $\sigma$ cuspidales (non nécessairement unitaires). On obtient donc pour toute représentation cuspidale de $\operatorname{GL}(a, E)$, $\sigma$, une égalité de traces :

$(1-3) \operatorname{tr} \tilde{\pi}(\sigma, \theta(\sigma))(h \theta)$

$$
\begin{aligned}
& =\sum_{\left\langle H=U\left(n_{1}\right) \times U\left(n_{2}\right)\right\rangle} \sum_{h_{H}^{\prime}} \Delta^{\left\langle U\left(n_{1}-2 a\right) \times U\left(n_{2}\right)\right\rangle}\left(h_{H}^{\prime}, h \theta\right) \operatorname{tr} \Pi^{H}(\sigma, 1) \\
& \quad+\sum_{\left\langle H=U\left(n_{1}\right) \times U\left(n_{2}\right)\right\rangle} \sum_{h_{H}^{\prime}} \Delta^{\left\langle U\left(n_{1}\right) \times U\left(n_{2}-2 a\right)\right\rangle}\left(h_{H}^{\prime}, h \theta\right) \operatorname{tr} \Pi^{H}(\omega \otimes \sigma, 2) .
\end{aligned}
$$

On généralise ce résultat en remplaçant $\tilde{\pi}$ par une somme avec coefficients de représentations.

Notations commodes pour les modules de Jacquet. Il sera commode d'écrire

$$
\tilde{\pi}(\sigma, \theta(\sigma))(h \theta)=: \operatorname{Jac}_{\sigma}^{\theta} \tilde{\pi} .
$$

Pour $\tau$ une représentation de $U(n, E / F)$ et pour $\sigma$ une représentation cuspidale comme ci-dessus, on écrira $\mathrm{Jac}_{\sigma} \tau$, ce qui est écrit ci-dessus $\tau(\sigma, 1)$.

\section{Appartenance à un paquet stable}

On fait d'abord une remarque générale. Soit $\tau^{\prime}$ une série discrète d'un groupe $U\left(m^{\prime}, E / F\right)$. On note $f_{\tau^{\prime}}$ l'élément de $I_{\text {cusp }}\left(U\left(m^{\prime}, E / F\right)\right.$ qui correspond à $\tau^{\prime}$; la projection de $I_{\text {cusp }}\left(U\left(m^{\prime}, E / F\right)\right)$ sur $I_{\text {cusp }}^{\text {st }}\left(U\left(m^{\prime}, E / F\right)\right)$ (par exemple suivant [Arthur 1996, 3.5]) n'annule pas $f_{\tau^{\prime}} ;$ l'argument m'a été donné par Waldspurger. 
On regarde les germes du caractère au voisinage de l'origine ; ils se développent par degré d'homogénéité et le degré formel est l'un de ces termes. Ce terme est stable et sa projection stable est donc non nulle et il y a donc un germe de la projection de $f_{\tau^{\prime}}$ qui est non nul. D'où la non nullité de la projection de $f_{\tau^{\prime}}$ sur $I_{\text {cusp }}^{\text {st }}(U(n, E / F)$.

\section{Point de réductibilité des induites de cuspidales pour les groupes unitaires}

3.1. Intégralité. Soit $\tau$ une représentation cuspidale de $U(n, E / F)$ et soient $\rho$ une représentation cuspidale irréductible $\theta$-invariante de $\operatorname{GL}\left(d_{\rho}, E\right)$. Soit $x \in \mathbb{R}_{>0}$ tel que l'induite $\rho||^{x} \times \tau$ soit réductible.

Proposition. Avec les notations précédentes, le réel $x$ est un demi-entier ou encore $2 x \in \mathbb{N}$.

Comme l'induite $\rho||^{x} \times \tau$ est réductible, il existe $\tau^{\prime}$ une sous-représentation de cette induite qui est une série discrète. Le module de Jacquet cuspidal de $\tau^{\prime}$ est réduit à l'unique terme $\rho||^{x} \otimes \tau$. On note $\Pi^{\prime}$ un paquet stable de représentations contenant $\tau^{\prime}$ et $\Sigma$ la représentation virtuelle image de $\Pi^{\prime}$ dans la décomposition de $I_{\text {cusp }}\left(\tilde{G}_{n+2 d_{\rho}}\right)$. On calcule les modules de Jacquet des 2 membres et on projette sur la représentation cuspidale $\left.\rho\right|^{x}$ de $\operatorname{GL}\left(d_{\rho}, E\right)$ en suivant (1-3). Le seul groupe endoscopique intervenant ici est le $U\left(n+2 d_{\rho}\right)$-stable par construction mais ici $\Sigma$ est une représentation virtuelle. Quand on calcule les modules de Jacquet de $\Pi^{\prime}$, le terme $\rho||^{x} \otimes \tau$ ne peut disparaître; en effet par réciprocité de Frobenius un tel terme ne peut provenir que de $\tau^{\prime}$; ainsi il existe une représentation $\sigma^{\prime} \in \Sigma^{\prime}$ telle que

$$
\operatorname{Jac}_{\rho||^{x}}^{\theta}\left(\sigma^{\prime}\right)=\sigma^{\prime}\left[\rho||^{x}, \rho||^{-x}\right] \neq 0 .
$$

On connaît la forme de $\sigma^{\prime}$ c'est une induite de Steinberg de la forme

$$
\sigma^{\prime}=\underset{\left(\rho^{\prime}, a^{\prime}\right) \in \mathscr{E}^{\prime}}{\times} \operatorname{St}\left(\rho^{\prime}, a^{\prime}\right) .
$$

La non nullité de (3-1) entraîne qu'il existe $\left(\rho^{\prime}, a^{\prime}\right) \in \mathscr{E}^{\prime}$ tel que $\rho^{\prime}=\rho$ et $x=$ $\left(a^{\prime}-1\right) / 2$. D'où la proposition.

\section{Finitude}

Soit $\tau$ une représentation cuspidale de $U(n, E / F)$; on note $\operatorname{Red}(\tau)$ l'ensemble des couples $\left(\rho, x_{\rho, \tau}\right)$ tels que $\rho$ soit une représentation cuspidale irréductible d'un groupe $\operatorname{GL}\left(d_{\rho}, E\right), \theta$ invariante et $x_{\rho, \tau}$ soit un demi-entier strictement supérieur à $1 / 2$ tels que l'induite :

$$
\rho||^{x_{\rho, \tau}} \times \tau
$$

soit réductible. D'après [Silberger 1980], $x_{\rho, \tau}$ quand $\rho$ et $\tau$ sont fixés est uniquement déterminé. 
Proposition. L'ensemble $\operatorname{Red}(\tau)$ est fini et on a l'inégalité

$$
\sum_{\left(\rho, x_{\rho, \tau} \in \operatorname{Red}(\tau)\right.}\left(2 x_{\rho, \tau}-1\right) d_{\rho} \leq n
$$

Ceci est analogue à un résultat de [Mœglin 2003] démontré par d'autres méthodes ; on améliorera l'inégalité dans la suite (de façon conforme à [Mœglin 2003]). Il suffit de montrer que l'inégalité de la proposition est vraie pour tout sous-ensemble fini de $\operatorname{Red}(\tau)$. On fixe $\mathscr{L}$ un sous-ensemble fini de $\operatorname{Red}(\tau)$ et on considère $\tau \mathscr{X}$ l'unique sous-module irréductible de l'induite :

$$
\left.\underset{\left(\rho, x_{\rho, \tau}\right) \in \mathscr{X}}{\times} \rho\right|^{x_{\rho, \tau}} \times \tau
$$

On peut ordonner $\mathscr{X}$ comme on veut ci-dessus sans changer le résultat et $\tau_{\mathscr{C}}$ est caractérisé par le fait que son module de Jacquet cuspidal est réduit à l'ensemble des termes :

$$
\bigotimes_{\left(\rho, x_{\rho, \tau}\right) \in \mathscr{L}} \rho||^{x_{\rho, \tau}} \otimes \tau
$$

où cette fois on considère tous les ordres possibles sur $\mathscr{X}$. On met $\tau_{\mathscr{L}}$ dans un paquet stable de représentations elliptiques et on considère l'image dans $I_{\text {cusp }}\left(\tilde{G}_{n_{\mathscr{L}}}\right)$ pour

$$
n_{\mathscr{X}}:=n+\sum_{\left(\rho, x_{\rho, \tau}\right) \in \mathscr{X}} d_{\rho} .
$$

On calcule les modules de Jacquet successifs en utilisant les représentations cuspidales $\left.\rho\right|^{x_{\rho, \tau}}$ associées aux éléments de $\mathscr{X}$; pour faire cela, on fixe un ordre sur $\mathscr{X}$ d'où un terme dans le module de Jacquet de $\tau_{\mathscr{L}}$ du type (4-1). On vérifie que les seules représentations elliptiques de $U\left(n_{\mathscr{X}}, E / F\right)$ qui contiennent ce terme comme sous-quotient de leur module de Jacquet sont précisément $\tau_{\mathscr{X}}$. Ainsi $\tau$ est obtenu dans le calcul des modules de Jacquet successifs. Et il existe une représentation elliptique de $\tilde{G}_{n_{\mathscr{X}}}$, $\tilde{\pi}_{\mathscr{C}}$ dont les modules de Jacquets successifs calculés comme expliqué en (1-3) sont non nuls. On écrit :

$$
\tilde{\pi}_{\mathscr{X}}=: \underset{\left(\rho^{\prime}, a^{\prime}\right) \in \mathscr{E}^{\prime}}{\times} \operatorname{St}\left(\rho^{\prime}, a^{\prime}\right) .
$$

La non nullité et le fait que les cuspidales intervenant dans $\mathscr{X}$ sont toutes différentes, entraînent qu'il existe un sous-ensemble $\mathscr{E}^{\prime \prime}$ de $\mathscr{E}^{\prime}$ tel que

$$
\mathscr{X}=\left\{\left(\rho^{\prime},\left(a^{\prime}-1\right) / 2\right) ;\left(\rho^{\prime}, a^{\prime}\right) \in \mathscr{E}^{\prime \prime}\right\} .
$$


On a $n_{\mathscr{L}}=\sum_{\left(\rho^{\prime}, a^{\prime}\right) \in \mathscr{C}^{\prime}} a^{\prime} d_{\rho^{\prime}}$ tout simplement parce que $\tilde{\pi}_{\mathscr{L}}$ est une représentation de $\tilde{G}_{n \mathscr{x}}$. Mais le module de Jacquet calculé donne une représentation de $\tilde{G}_{n}$, d'où

$$
n=n_{\mathscr{X}}-\sum_{\left(\rho^{\prime}, a^{\prime}\right) \in \mathscr{C}^{\prime \prime}} 2 d_{\rho^{\prime}}=\sum_{\left(\rho^{\prime}, a^{\prime}\right) \in \mathscr{C}^{\prime \prime}} 2 d_{\rho^{\prime}}\left(a^{\prime}-3\right) / 2+\sum_{\left(\rho^{\prime}, a^{\prime}\right) \in \mathscr{C}^{\prime}-\mathscr{C}^{\prime \prime}} a^{\prime} d_{\rho^{\prime}} .
$$

Pour $\left(\rho^{\prime}, a^{\prime}\right) \in \mathscr{C}^{\prime \prime}$, on a $2\left(a^{\prime}-3\right) / 2=\left(a^{\prime}-1\right)-1=2 x_{\rho, \tau}-1$. D'où encore

$$
n=\sum_{\left(\rho, x_{\rho, \tau}\right) \in \mathscr{X}} d_{\rho}\left(2 x_{\rho, \tau}-1\right)+\sum_{\left(\rho^{\prime}, a^{\prime}\right) \in \mathscr{C}^{\prime}} a^{\prime} d_{\rho^{\prime}} .
$$

Cela donne a fortiori l'inégalité cherchée et démontre la proposition.

4.1. Propriétés générales des séries discrètes et représentations elliptiques. Ici on fixe $n$ et $\tau$ une représentation tempérée. Par des méthodes totalement standard, on montre qu'il existe une représentation cuspidale $\tau_{0}$ d'un groupe $U\left(m_{0}, E / F\right)$ avec $m_{0} \leq m$ et un ensemble totalement ordonné de segments à la Zelevinski $\left(\rho^{\prime}, a^{\prime}, b^{\prime}\right) \in \mathscr{F}$, où $\rho^{\prime}$ est une représentation cuspidale unitaire de $\operatorname{GL}\left(d_{\rho^{\prime}}, E\right)$, et les $a^{\prime}, b^{\prime}$ sont des réels tel que $a^{\prime}-b^{\prime}+1 \in \mathbb{N}$ avec une inclusion :

$$
\tau \hookrightarrow \underset{\left(\rho^{\prime}, a^{\prime}, b^{\prime}\right) \in \mathscr{F}}{\times}\left\langle\rho||_{E}^{a^{\prime}}, \rho||_{E}^{b^{\prime}}\right\rangle \times \tau_{0} .
$$

Ici je prends comme convention que $\left\langle\rho||_{E}^{a^{\prime}}, \rho||_{E}^{b^{\prime}}\right\rangle$ est la représentation

$$
\operatorname{St}\left(\rho^{\prime}, a^{\prime}-b^{\prime}+1\right)||^{\left(a^{\prime}+b^{\prime}\right) / 2} \text {. }
$$

Pour écrire cela, on n'utilise rien sur $\tau$ sauf que c'est une représentation irréductible. Rappelons quand même rapidement la démonstration ; on fixe d'abord un ensemble $\mathscr{F}^{\prime}$, totalement ordonné, formé de couples $\rho^{\prime}, y^{\prime}$ tel que $\rho^{\prime}$ soit une représentation cuspidale unitaire et $y^{\prime}$ un réel et tel que l'on ait une inclusion :

$$
\tau \hookrightarrow \underset{\left(\rho^{\prime}, y^{\prime}\right) \in \mathscr{F}^{\prime}}{\times} \rho^{\prime}||_{E}^{y^{\prime}} \times \tau_{0} .
$$

On fixe un tel choix. Ensuite on fixe $\left(\rho_{0}, y_{0}\right)$ tel que $y_{0}$ soit minimum et on pousse $\rho_{0}||_{E}^{y_{0}}$ vers la gauche; par exemple sans changer l'ensemble $\mathscr{F}^{\prime}$ on change son ordre tel que (4-3) soit toujours satisfait mais $\left(\rho_{0}, y_{0}\right)$ est le plus "petit" possible (le plus à gauche possible). Cela permet de remplacer l'induite formée par les représentations qui le précèdent et lui même par un segment comme ci-dessus. On obtient alors la propriété suivante supplémentaire sur $\mathscr{E}$ :

(4-4) si $\left(\rho^{\prime \prime}, a^{\prime \prime}, b^{\prime \prime}\right)<\left(\rho^{\prime}, a^{\prime}, b^{\prime}\right)$ avec $\rho^{\prime} \simeq \rho^{\prime \prime}$ pour l'ordre de $\mathscr{E}$, alors $b^{\prime \prime} \leq b^{\prime}$.

On peut obtenir une propriété de plus quand on part d'un ensemble $\mathscr{g}^{\prime}$ tel que parmi tous les choix possibles, le nombre de $y^{\prime}<0$ soit maximal pour le choix fait. Cette propriété de $\mathscr{g}^{\prime}$ entraîne : soit $\left(\rho^{\prime}, a^{\prime}, b^{\prime}\right) \in \mathscr{F}$ tel que $b^{\prime}>0$, alors il existe 
$\left(\rho^{\prime}, a^{\prime \prime}, b^{\prime \prime}\right) \in \mathscr{g}$ (éventuellement le même élément) avec $a^{\prime \prime} \geq a^{\prime}$ et $\rho^{\prime}||^{b^{\prime \prime}} \times \tau_{0}$ est réductible.

Si on utilise en plus l'hypothèse que $\tau$ est une représentation tempérée, on va vérifier que si $\mathscr{F}$ vérifie (4-2) et (4-4) alors pour tout $\left(\rho^{\prime}, a^{\prime}, b^{\prime}\right) \in \mathscr{F}$ nécessairement $a^{\prime}+b^{\prime} \geq 0$.

En effet supposons qu'il existe $\left(\rho^{\prime}, a^{\prime}, b^{\prime}\right) \in \mathscr{J}$ tel que $a^{\prime}+b^{\prime}<0$; on fixe un tel triplet de tel sorte que $a^{\prime}+b^{\prime}$ soit minimal. Sans changer $\mathscr{F}$ on fixe un ordre sur $\mathscr{F}$ tel que (4-2) soit encore vrai et le cas particulier de (4-4) quand on fixe notre $\left(\rho, a^{\prime}, b^{\prime}\right)$ et $\left(\rho^{\prime}, a^{\prime}, b^{\prime}\right)$ soit le plus "petit" possible pour cet ordre, il est plus simple de dire le plus à gauche possible dans (4-2). On va vérifier que $\left(\rho^{\prime}, a^{\prime}, b^{\prime}\right)$ est nécessairement le premier élément de $\mathscr{F}$. En effet soit $\left(\rho^{\prime \prime}, a^{\prime \prime}, b^{\prime \prime}\right) \in \mathscr{J}$ précédant $\left(\rho, a^{\prime}, b^{\prime}\right)$. Si la représentation induite :

$$
\left\langle\rho^{\prime \prime}, a^{\prime \prime}, b^{\prime \prime}\right\rangle \times\left\langle\rho^{\prime}, a^{\prime}, b^{\prime}\right\rangle
$$

est irréductible, on peut échanger les 2 facteurs sans perdre l'inclusion en (4-2) et le cas particulier de (4-4) qui nous intéresse. Donc par minimalité sur l'ordre de $\mathscr{g}$, cette induite est réductible d'où $\rho^{\prime \prime} \simeq \rho^{\prime}$ et les segments $\left[a^{\prime \prime}, b^{\prime \prime}\right],\left[a^{\prime}, b^{\prime}\right]$ sont liés; rappelons que ce sont des segments décroissants et que l'on a $b^{\prime \prime} \leq b^{\prime}$ donc la liaison entraîne aussi $\left.\left.a^{\prime \prime} \in\right] a^{\prime}, b^{\prime}-1\right]$ et $b^{\prime \prime}<b^{\prime}$. D'où $a^{\prime \prime}+b^{\prime \prime}<a^{\prime}+b^{\prime}$ ce qui contredit la minimalité de $\left(\rho^{\prime}, a^{\prime}, b^{\prime}\right)$. On vérifie alors que l'exposant du module de Jacquet de $\tau$ donné par réciprocité de Frobenius et (4-2) n'est pas dans le cône positif obtus fermé comme il devrait l'être (critère de Casselman) d'où la contradiction cherchée. Pour les séries discrètes les inégalités sont strictes.

\section{Changement de base réciproque des représentations cuspidales}

Notre approche du changement de base se fait en utilisant les propriétés de réductibilité des induites de cuspidales ; ces propriétés sont précisément controlées par des pôles de fonction $L$; c'est la théorie d'Harish-Chandra amplifiée par Shahidi.

5.1. Support cuspidal étendu d'une série discrète de $U(n, E / F)$. En 3.1, on a montré que les points de réductibilité des induites de cuspidales sont demi-entiers : précisément soit $\tau$ une représentation cuspidale de $U\left(n_{0}, E / F\right)$ (ici $n_{0}$ peut être $0)$; soit $\rho$ une représentation cuspidale irréductible d'un $\operatorname{GL}\left(d_{\rho}, E\right)$ et soit $x$ un réel strictement positif tel que $\rho \|^{x} \times \tau_{0}$ soit réductible. Alors il existe $a$ un entier naturel tel que $x=(a-1) / 2$. Il faut remarquer que le cas $n_{0}=0$ est dû à Shahidi [1990].

On a aussi montré que pour un bon choix d'ensemble $\mathscr{E}$, on a l'inclusion (4-2), c'est-à-dire

$$
\tau \hookrightarrow \underset{\left(\rho^{\prime}, a^{\prime}, b^{\prime}\right) \in \mathscr{F}}{\times}\left\langle\rho, a^{\prime}, b^{\prime}\right\rangle \times \tau_{0} .
$$


On va vérifier encore que pour tout $\left(\rho^{\prime}, a^{\prime}, b^{\prime}\right) \in \mathscr{F}, a^{\prime}, b^{\prime}$ sont des demi-entiers.

Une telle assertion est évidemment indépendante du choix de $\mathscr{F}$, c'est une propriété de "demi-intégralité" du support cuspidal de $\tau$. C'est élémentaire et on en rappelle la démonstration :

On fixe $\mathscr{S}$ un ensemble totalement ordonné comme en 4.1 vérifiant (4-2) et (4-4). On impose en plus la propriété de minimalité

$$
\sum_{\left(\rho^{\prime}, a^{\prime}, b^{\prime}\right) \in \mathscr{F}} \sum_{x \in\left[a^{\prime}, b^{\prime}\right]} x
$$

est minimal parmi les choix de $\mathscr{F}$ possible. Ceci est tout à fait compatible aux propriétés requises. Supposons qu'il existe $\left(\rho^{\prime}, a^{\prime}, b^{\prime}\right) \in \mathscr{F}$ avec $a^{\prime}, b^{\prime}$ non demientiers. On peut supposer que cet élément est le plus à droite possible. On peut le faire commuter avec tous les éléments plus à droite que lui car ceux là font intervenir des demi-entiers et comme les points de réductibilité des induites de cuspidales sont des demi-entiers, on obtient l'isomorphisme :

$$
\left\langle\rho^{\prime}, a^{\prime}, b^{\prime}\right\rangle \times \tau_{0} \simeq\left\langle\rho^{\prime},-b^{\prime},-a^{\prime}\right\rangle \times \tau_{0} .
$$

Or on sait que $a^{\prime}+b^{\prime} \geq 0$ et $a^{\prime}-b^{\prime}+1 \in \mathbb{N}$ (car $\left[a^{\prime}, b^{\prime}\right]$ est un segment décroissant). D'où nécessairement $a^{\prime}+b^{\prime}>0$ et $\sum_{x \in\left[a^{\prime}, b^{\prime}\right]} x>\sum_{x^{\prime} \in\left[-b^{\prime},-a^{\prime}\right]} x^{\prime}$. En ayant changé $\left(a^{\prime}, b^{\prime}\right)$ en $\left(-b^{\prime},-a^{\prime}\right)$, on va trouver un nouvel ensemble qui donnera un (5-1) plus petit que pour $\mathscr{F}$. Ceci est donc en contradiction avec la minimalité fixée.

5.2. Préliminaire sur le support cuspidal étendu. Soit $\tau$ une représentation cuspidale de $U(n, E / F)$ et soit $\rho$ une représentation cuspidale irréductible $\theta$ invariante de $\operatorname{GL}\left(d_{\rho}, E\right)$. On suppose qu'il existe $x>1 / 2$ tel que l'induite $\rho||_{E}^{x} \times \tau$ soit réductible; on sait d'après [Silberger 1980] que $x$ est alors uniquement déterminé et on pose $a_{\rho, \tau}:=2 x-1$. On sait maintenant que $a_{\rho, \tau}$ est un entier.

Lemme. Soit $\ell$ un demi-entier dans $\left[1 / 2,\left(a_{\rho, \tau}+1\right) / 2\right]$ tel que $2 \ell+1$ soit de même parité que $a_{\rho, \tau}$. Alors l'induite:

$$
\rho||_{E}^{\ell} \times \cdots \times \rho \mid \|_{E}^{\left(a_{\rho, \tau}+1\right) / 2} \times \tau
$$

a un unique sous-module irréductible et ce sous-module est une série discrète.

On note $m^{\prime}$ l'entier $\left.n+2\left(a_{\rho, \tau}+1\right) / 2-\ell+1\right) d_{\rho}$. La représentation induite écrite dans l'énoncé est une représentation de $U\left(m^{\prime}, E / F\right)$. On note $\sigma$ cette représentation induite. L'unicité du sous-module irréductible résulte de la réciprocité de Frobenius : soit $\tau_{0}$ un sous-module irréductible. On a $\operatorname{Hom}_{U\left(m^{\prime}, E / F\right)}\left(\tau_{0}, \sigma\right) \neq 0$ entraîne que le module de Jacquet de $\tau_{0}$ admet le terme

$$
\left.\rho||_{E}^{\ell} \otimes \cdots \otimes \rho\right|^{\left(a_{\rho, \tau}+1\right) / 2} \otimes \tau
$$


comme quotient. Le terme que l'on vient d'écrire intervient avec multiplicité 1 comme sous-quotient du module de Jacquet de $\sigma$ et comme prendre les modules de Jacquet est un foncteur exact, $\tau_{0}$ est unique et intervient avec multiplicité 1 comme sous-quotient de $\sigma$.

On précise d'abord le lemme en annonçant qu'en plus le module de Jacquet cuspidal de $\tau_{0}$ est réduit au terme (5-2). On démontre alors cette version plus forte du lemme par récurrence descendante sur $\ell$. Le résultat est vrai pour $\ell=\left(a_{\rho, \tau}+1\right) / 2$. On suppose donc $\ell<\left(a_{\rho, \tau}+1\right) / 2$; on change la notation $\tau_{0}$ précédente en $\tau_{\ell}$ pour la faire dépendre de $\ell$. On considère à l'intérieur de $\sigma$ l'intersection des deux sous-modules

$$
\rho||_{E}^{\ell} \times \tau_{\ell+1} \cap\left\langle\rho, \ell,\left(a_{\rho, \tau}+1\right) / 2\right\rangle \times \tau,
$$

où $\left\langle\rho, \ell,\left(a_{\rho, \tau}+1\right) / 2\right\rangle$ est l'unique sous-module irréductible pour le groupe linéaire convenable de l'induite :

$$
\rho \|\left.\right|_{E} ^{\ell} \times \cdots \times \rho||_{E}^{\left(a_{\rho, \tau}+1\right) / 2} .
$$

Par unicité de $\tau_{\ell}$ l'intersection contient $\tau_{\ell}$. On calcule les modules de Jacquet de chacune des induites écrites le module de Jacquet de $\tau_{\ell}$ est inclus dans l'intersection des modules de Jacquet. Il suffit donc de vérifier que cette intersection est réduite au terme (5-2). On l'écrit explicitement pour $\ell=\left(a_{\rho, \tau}-1\right) / 2$, le cas général suit la même ligne. Le module de Jacquet de $\left.\rho\right|_{E} ^{\ell} \times \tau_{\ell+1}$ a pour semi-simplifié la somme des quatres termes (on pose $a:=a_{(\rho, \tau)}$ ) et on oublie l'indice $E$ :

$$
\begin{aligned}
\left(\rho \|\left.\right|^{(a-1) / 2}\right. & \left.\otimes \rho \|^{(a+1) / 2} \otimes \tau\right) \oplus\left(\rho\left\|^{(a+1) / 2} \otimes \rho\right\|^{(a-1) / 2} \otimes \tau\right) \\
& \oplus\left(\rho\left\|\left.\right|^{-(a-1) / 2} \otimes \rho\right\|^{(a+1) / 2} \otimes \tau\right) \oplus\left(\rho\left\|^{(a+1) / 2} \otimes \rho\right\|^{-(a-1) / 2} \otimes \tau\right) .
\end{aligned}
$$

Le module de Jacquet de l'induite $\left\langle\rho, \ell,\left(a_{\rho, \tau}+1 / 1\right)\right\rangle \times \tau$ est constitué des 4 termes :

$$
\begin{aligned}
\left(\rho \|\left.\right|^{(a-1) / 2}\right. & \left.\otimes \rho \|^{(a+1) / 2} \otimes \tau\right) \oplus\left(\rho\left\|^{(a-1) / 2} \otimes \rho\right\|^{-(a+1) / 2} \otimes \tau\right) \\
& \oplus\left(\left.\rho\left\|^{-(a+1) / 2} \otimes \rho\right\|\right|^{(a-1) / 2} \otimes \tau\right) \oplus\left(\rho\left\|^{-(a+1) / 2} \otimes \rho\right\|^{-(a-1) / 2} \otimes \tau\right) .
\end{aligned}
$$

D'où le résultat dans ce cas particulier. Ce qui fait "marcher" la machine en général est que le module de Jacquet de la première induite a dans tous ses termes $\rho||^{(a+1) / 2}$ tandis que la deuxième, a dans tous ses termes hormis le terme (5-2) $\rho||^{-(a+1) / 2}$. L'intersection est donc réduite à (5-2).

\subsection{Support cuspidal étendu des représentations cuspidales des groupes uni- taires.}

Definition. Soit $\tau$ une représentation cuspidale irréductible de $U(m, E / F)$. Le support cuspidal étendu de $\tau$ est, par définition, le support cuspidal pour le groupe 
linéaire convenable de la représentation

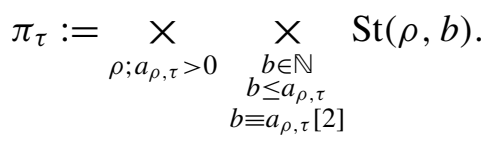

Proposition. (i) $\pi_{\tau}$ est une représentation de $\operatorname{GL}(n, E)$.

(ii) Soit $\tilde{\pi}$ une représentation elliptique de $\tilde{G}_{n}$ dont la composante sur

$$
I_{\text {cusp }}^{\langle U(n), \mathrm{st}\rangle}(U(n, E / F))
$$

contient $\tau$ de façon non nulle; alors $\tilde{\pi}$ est un prolongement de $\pi_{\tau}$.

On note $n^{\prime}$ l'entier tel que $\pi_{\tau}$ soit une représentation de $\operatorname{GL}\left(n^{\prime}, E\right)$. On montre d'abord que $n^{\prime} \leq n$ en reprenant la démonstration de la proposition du paragraphe 4 .

Il faut généraliser la définition de $\tau_{\ell}$ de 5.2 pour faire intervenir toutes les représentations $\rho$ telles que $a_{\rho, \tau}>0$; cet ensemble est fini grâce à la même proposition. On montre donc qu'il existe une série discrète irréductible $\tau^{\prime}$ dont le module de Jacquet contient le terme :

$$
\bigotimes_{\rho ; a_{\rho, \tau}>0}\left(\left.\rho\right|^{\delta_{\rho}} \otimes \cdots \otimes \rho \|\left.\right|^{\left(a_{\rho, \tau}+1\right) / 2}\right) \otimes \tau,
$$

où $\delta_{\rho}$ vaut 1 si $a_{\rho, \tau}$ est impair et $1 / 2$ sinon. Le module de Jacquet de $\tau^{\prime}$ n'est pas irréductible parce que l'on peut faire commuter des termes correspondant à des cuspidales différentes mais cela est la seule opération possible. On note $m^{\prime}$ l'entier tel que $\tau^{\prime}$ soit une représentation de $U\left(m^{\prime}, E / F\right)$.

On va aussi avoir besoin de la propriété d'unicité suivante : soit $\tau^{\prime \prime}$ une représentation elliptique de $U\left(m^{\prime}, E / F\right)$ dont le module de Jacquet contient comme sousquotient un des termes du type (5-3), alors $\tau^{\prime \prime}=\tau^{\prime}$. En effet on applique la construction de 4.1 et on écrit une inclusion :

$$
\tau^{\prime \prime} \hookrightarrow \underset{\left(\rho^{\prime}, a^{\prime}, b^{\prime}\right) \in \mathscr{\mathcal { F }}}{\times}\left\langle\rho^{\prime}, a^{\prime}, b^{\prime}\right\rangle \times \tau,
$$

où les notations sont celles de loc.cite et $\tau$ est nécessairement la représentation cuspidale fixée. En comparant les supports cuspidaux ordinaires, on vérifie déjà que pour tout $\left(\rho^{\prime}, a^{\prime}, b^{\prime}\right) \in \mathscr{J}, b^{\prime}>0$; en effet il n'y a pas de multiplicité dans le support cuspidal. Il faut maintenant vérifier les points suivants : soit $\rho^{\prime}$ fixé et $\left(\rho^{\prime}, a^{\prime}, b^{\prime}\right) \in \mathscr{J}$. On suppose d'abord que $\left(\rho^{\prime}, a^{\prime}, b^{\prime}\right)$ ne précède aucun autre élément de $\mathscr{F}$ de la forme $\left(\rho^{\prime}, a^{\prime \prime}, b^{\prime \prime}\right)$ alors $b^{\prime}=\left(a_{\rho, \tau}+1\right) / 2$. S'il n'en était pas ainsi on pourrait remplacer $b^{\prime}$ par $-b^{\prime}$ et contredire la positivité déjà démontrée. Ainsi pour cet élément on a aussi $a^{\prime}=b^{\prime}$; puis on montre progressivement que si $\left(\rho^{\prime}, a^{\prime}, b^{\prime}\right)$ précède $t$ éléments de $\mathscr{F}$ de la forme $\left(\rho^{\prime}, a^{\prime \prime}, b^{\prime \prime}\right)$ alors $b^{\prime}=\left(a_{\rho, \tau}+1\right) / 2-t: b^{\prime}$ ne peut être plus grand car cela contredirait le fait que le support cuspidal ordinaire 
n'a pas de multiplicité (avec un argument par récurrence sur $t$ ) ni plus petit car sinon on pourrait remplacer $b^{\prime}$ par $-b^{\prime}$ après avoir fait commuter et contredire la positivité déjà prouvée; puis on obtient $a^{\prime}=b^{\prime}$ simplement comme conséquence de la connaissance du support cuspidal ordinaire (en termes imagés, tous les $\rho^{\prime}||^{x}$ avec $x>b^{\prime}$ ont déjà été utilisés). Cela prouve l'unicité cherchée.

On considère la projection de $\tau^{\prime}$ sur $I_{\text {cusp }}^{\text {st }}\left(U\left(m^{\prime}, E / F\right)\right)$; cela donne une représentation virtuelle $\omega^{\prime}$ qui contient de façon non nulle $\tau^{\prime}$. Et on considère l'image de cet élément stable dans $I_{\text {cusp }}\left(\tilde{G}_{m^{\prime}}\right)$. Cela fournit une combinaison linéaire de représentations à laquelle on peut appliquer (1-3). On calcule successivement les modules de Jacquet pour les représentations cuspidales apparaissant dans (5-3) à gauche de $\tau$ dans l'ordre écrit en commençant pas la gauche. Le calcul du module de Jacquet pour $\omega^{\prime}$ est une combinaison linéaire de termes mais le terme (5-3) ne peut pas disparaître car il n'appartient qu'à $\tau^{\prime}$ d'après l'unicité ci-dessus. Ainsi en appliquant des $\mathrm{Jac}^{\theta}$ convenables au membre de gauche de (1-3) on obtient aussi un terme non nul. Soit donc $\Omega$ une représentation de $\tilde{G}_{m^{\prime}}$ qui donne un terme non nul quand on prend ces modules de Jacquet. On sait par hypothèse que $\Omega$ est elliptique donc de la forme :

$$
\Omega=\underset{\left(\rho^{\prime}, b^{\prime}\right) \in \mathscr{E}}{\times} \operatorname{St}\left(\rho^{\prime}, b^{\prime}\right)
$$

où $\mathscr{E}$ est un ensemble convenable de couples. Le module de Jacquet est facile à calculer. Il ne contient pas le terme voulu si $\mathscr{E}$ ne contient pas les couples

$$
\mathscr{E}^{\prime}:=\bigcup_{\rho ; a_{\rho, \tau}>0} \bigcup_{b \leq a ; b \geq 0, b \equiv a[2]}(\rho, b+2) .
$$

Le module de Jacquet cherché est alors

$$
\pi^{\prime}:=\underset{\left(\rho^{\prime}, b^{\prime}\right) \in \mathscr{C}^{\prime}}{\times} \operatorname{St}\left(\rho^{\prime}, b^{\prime}-2\right) \underset{\left(\rho^{\prime}, b^{\prime}\right) \in \mathscr{E}-\mathscr{C}^{\prime}}{\times} \operatorname{St}\left(\rho^{\prime}, b^{\prime}\right) .
$$

Or $\pi_{\tau}$ n'est autre que $\times_{\left(\rho^{\prime}, b^{\prime}\right) \in_{\mathscr{C}^{\prime}}} \operatorname{St}\left(\rho^{\prime}, b^{\prime}-2\right)$. D'où $n^{\prime} \leq n$.

Montrons que $n^{\prime} \geq n$ : on fixe $\tilde{\pi}$ et on lui associe un élément de $I_{\text {cusp }}\left(\tilde{G}_{n}\right)$. On suppose que dans la décomposition de 1.4 la composante de cet élément fait intervenir $\tau$ de façon non nulle. C'est possible. On écrit

$$
\pi:=\underset{(\rho, b) \in \mathscr{F}}{\times} \operatorname{St}(\rho, b)
$$

pour des choix convenables. On fixe $\rho$ tel qu'il existe $b$ avec $(\rho, b) \in \mathscr{F}$ et on fixe même $b_{0}$ maximal avec cette propriété. On a alors $a_{\rho, \tau}=b_{0}$ : en effet on considère la représentation :

$$
\pi\left(\psi^{\prime}\right):=\underset{\left(\rho^{\prime}, b^{\prime}\right) \in \mathscr{q}-\left\{\left(\rho, b_{0}\right)\right\}}{\times} \operatorname{St}\left(\rho^{\prime}, b^{\prime}\right) \times \operatorname{St}\left(\rho, b_{0}+2\right)
$$


un prolongement de cette représentation à $\tilde{G}_{n+2 d_{\rho}}$. C'est une représentation $\theta$ discrète (elliptique) par maximalité de $b_{0}$. Puis on considère le module de Jacquet

$$
\operatorname{Jac}_{\rho\|\|_{E}^{\theta}}^{\left(b_{0}+1\right) / 2}
$$

qui est un prolongement de $\pi(\psi)$. Dans la projection sur la partie stable, ce module de Jacquet fait intervenir $\tau$ ce qui entraîne que dans sa décomposition $\pi\left(\psi^{\prime}\right)$ fait intervenir soit dans $I_{\text {cusp }}^{\text {st }}\left(U\left(n+2 d_{\rho}, E / F\right)\right.$ un sous-quotient de l'induite

$$
\rho||_{E}^{\left(b_{0}+1\right) / 2} \times \tau
$$

soit dans $I_{\text {cusp }}^{U(n) \times U\left(2 d_{\rho}\right.}\left(U(n, E / F) \times U\left(2 d_{\rho}, E / F\right)\right.$ un sous-quotient de la représentation

$$
\tau \otimes\left(\text { ind } \omega \otimes \rho||^{\left(b_{0}+1\right) / 2}\right) .
$$

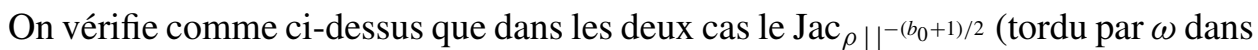
le deuxième cas) de ce sous-quotient est nécessairement 0 car $\operatorname{Jac}_{\rho||^{-\left(b_{0}+1\right) / 2}}^{\theta} \pi\left(\psi^{\prime}\right)$ s'annulle. D'où la réductibilité de l'induite $\left.\rho\right|^{\left(b_{0}+1\right) / 2} \times \tau$ dans le premier cas et de l'induite de $\omega \otimes \rho||_{E}^{\left(b_{0}+1\right) / 2}$ dans le deuxième cas. Le deuxième cas est alors exclu car $\left(b_{0}+1\right) / 2 \geq 1$ et par les points de réductibilité pour une telle induite sont soit 0 soit $1 / 2$. D'où le fait que $b_{0}=a_{\rho, \tau}$ comme annoncé. Il faut encore démontrer que si $\rho$ est fixé et qu'il existe $b, b^{\prime}$ des entiers avec $(\rho, b)$ et $\left(\rho, b^{\prime}\right)$ dans $\mathscr{g}$ alors $b$ et $b^{\prime}$ ont même parité. S'il n'en est pas ainsi on prend pour $b_{0}$ le plus grand entier tel que $\left(\rho, b_{0}\right) \in \mathscr{F}$ et $b_{0}$ de parité différente de $a_{\rho, \tau}$. On refait la démonstration ci-dessus car $\pi\left(\psi^{\prime}\right)$ est encore une représentation elliptique de $\tilde{G}_{n+2 d_{\rho}}$. Alors l'induite :

$$
\rho||_{E}^{\left(b_{0}+1\right) / 2} \times \tau
$$

serait encore réductible ce qui contredirait l'unicité des points de réductibilité dans $\mathbb{R}_{\geq 0}$ [Silberger 1980]. D'où la contradiction cherchée.

On a alors clairement

$$
\sum_{(\rho, b) \in \mathcal{F}} b d_{\rho}=n \leq \sum_{\substack{(\rho, b) \in \mathcal{F} \\ b \equiv a_{\rho, \tau} \\ b \leq a_{\rho, \tau}}} b d_{\rho}=n^{\prime} .
$$

En fait on démontre beaucoup plus, puisque l'égalité force que si $(\rho, b) \in \mathscr{J}$ avec $b>2$ alors $(\rho, b-2) \in \mathscr{F}$. En d'autres termes $\pi=\pi_{\tau}$. On a donc démontré aussi (ii).

5.4. Support cuspidal étendu pour les séries discrètes d'un groupe unitaire. On a défini en le support cuspidal étendu pour les représentations cuspidales des groupes unitaires. Soit maintenant $\tau$ une série discrète irréductible de $U(n, E / F)$. On revient à (4-2), d'où l'existence d'un ensemble de triplets $\left(\rho^{\prime}, a^{\prime}, b^{\prime}\right)$ tels que 
$\rho^{\prime}$ soit une représentation cuspidale irréductible $\theta$ invariante d'un groupe linéaire et $a^{\prime}, b^{\prime}$ un segment décroissant. On a montré que $a^{\prime}, b^{\prime}$ sont des demi-entiers; on a aussi la représentation cuspidale $\tau_{0}$ que l'on peut appeler comme en [Mœglin 2002] et [Mœglin et Tadić 2002] le support cuspidal partiel et on pose :

$$
\operatorname{Supp} \operatorname{cusp}(\tau)=\bigcup_{\left(\rho^{\prime}, a^{\prime}, b^{\prime}\right) \in \mathscr{\Phi}} \bigcup_{y \in\left[a^{\prime}, b^{\prime}\right]}\left\{\rho^{\prime}||_{E}^{y}, \rho^{\prime}||_{E}^{-y}\right\} \cup \operatorname{Supp} \operatorname{cusp}\left(\tau_{0}\right) .
$$

Cette définition ne dépend que du support cuspidal ordinaire de $\tau$ et est donc indépendante du choix de $\mathscr{F}$ : en effet écrivons $\tau$ comme sous-quotient d'une induite de la forme $\times_{\lambda \in \mathscr{S}} \lambda \times \tau_{0}$ où $\mathscr{Y}$ est un ensemble de représentations cuspidales irréductibles de groupes linéaires convenables et $\tau_{0}$ est une représentation cuspidale irréductible d'un groupe unitaire comme ci-dessus. Alors

$$
\operatorname{Supp} \operatorname{cusp}(\tau)=\bigcup_{\lambda \in \mathscr{S}}\{\lambda, \theta(\lambda)\} \cup \operatorname{Supp} \operatorname{cusp}\left(\tau_{0}\right) .
$$

La définition s'étend sans problème aux représentations tempérées puisqu'une représentation tempérée irréductible est un sous-module d'une induite de séries discrètes. On peut aussi vérifier qu'une représentation elliptique est combinaison linéaire de représentations tempérées ayant toutes même support cuspidal étendu puisque ces représentations sont toutes dans une même induite de séries discrètes. Ceci permet donc de définir aussi le support cuspidal étendu d'une représentation elliptique. Le support cuspidal d'une représentation tempérée fait intervenir des représentations cuspidales de la forme $\left.\rho\right|_{E} ^{x}$ où $\rho$ est unitaire non nécessairement $\theta$-invariante; on vérifie aisément que pour une représentation elliptique ceci ne peut pas se produire, $\rho$ est nécessairement $\theta$-invariant.

Lemme. Il existe une unique représentation tempérée de $\mathrm{GL}(n, E)$, $\pi_{\tau}$ telle que le support cuspidal de $\pi_{\tau}$ soit précisément $\operatorname{Supp} \operatorname{cusp}(\tau)$.

Avant de démontrer ce lemme remarquons que cela donne une limitation forte sur les ensembles de représentations cuspidales qui peuvent être un support cuspidal étendu d'une représentation tempérée de $U(n, E / F)$. En effet, une représentation tempérée de $\operatorname{GL}(n, E)$ est nécessairement de la forme

$$
\underset{(\rho, a) \in \mathscr{E}}{\times} \operatorname{St}(\rho, a),
$$

avec des notations que l'on espère évidentes et le support cuspidal de cette représentation n'est autre que

$$
\left.\bigcup_{(\rho, a) \in \mathscr{E}} \bigcup_{u \in\left[\frac{a-1}{2},-\frac{a-1}{2}\right]} \rho\right|_{E} ^{u} .
$$


Il suffit de démontrer le lemme pour $\tau$ une série discrète. Soit donc $\tau$ une série discrète.

Le support cuspidal étendu tel que défini est un ensemble de couples $\rho, x$ (écrit $\left.\rho||_{E}^{x}\right)$ où $\rho$ est une représentation cuspidale unitaire $\theta$-stable et $x$ un réel. Pour chaque $\rho$ fixée, l'ensemble des $x$ qui apparaît est un ensemble de demi-entiers stable par multiplication par -1 . Le lemme est équivalent à prouver que cet ensemble est une réunion de segments centrés en l'origine et il est clair que si ceci est vrai la décomposition en segments est uniquement déterminée ; une autre façon de dire les choses est que pour $\operatorname{GL}(n, E)$ les représentations tempérées sont uniquement déterminées par leur support cuspidal. Il n'y a donc que l'existence à démontrer.

Fixons $\left(\rho^{\prime}, a^{\prime}, b^{\prime}\right) \in \mathscr{F}$ et supposons que $b^{\prime} \leq 0$; alors $\bigcup_{y \in\left[a^{\prime}, b^{\prime}\right]}\left\{\rho^{\prime}||_{E}^{y}, \rho^{\prime}||_{E}^{-y}\right\}$ est le support cuspidal de la représentation $\operatorname{St}\left(\rho^{\prime}, 2 a^{\prime}+1\right) \times \operatorname{St}\left(\rho^{\prime},-2 b^{\prime}+1\right)$. Dans l'écriture (4-2), on peut remplacer l'induite :

$$
\underset{\left(\rho^{\prime}, a^{\prime}, b^{\prime}\right) \in \mathscr{q} ; b^{\prime}>0}{\times}\left\langle\rho^{\prime}, a^{\prime}, b^{\prime}\right\rangle \times \tau_{0}
$$

qui sont les termes les plus à droite par un sous-quotient $\tau^{\prime}$ convenable. Avec l'hypothèse loisible que l'on a construit $\mathscr{F}$ en partant d'un ensemble $\mathscr{F}^{\prime}$ avec un nombre maximum de $\rho^{\prime \prime}||_{E}^{y^{\prime \prime}}$ avec $y^{\prime \prime}<0$, on sait que le module de Jacquet de $\tau^{\prime}$ ne contient que des termes de la forme $\bigotimes_{\rho^{\prime \prime}, y^{\prime \prime}>0} \rho^{\prime \prime}||_{E}^{y^{\prime \prime}} \times \tau_{0}$. Ainsi $\tau^{\prime}$ est une série discrète et c'est ce que l'on a appelé en [Mœglin 2002] une série discrète fortement positive, on en a déjà parlé ci-dessus. Il est très facile de classifier ces séries discrètes quand on connaît les différents termes $a_{\rho, \tau_{0}}$. Pour ces séries discrètes, on démontre que pour toute $\rho$ représentation cuspidale $\theta$-invariante irréductible d'un $\operatorname{GL}\left(d_{\rho}, E\right)$ telle que $a_{\rho, \tau_{0}} \geq 0$, il existe un ensemble totalement ordonné, éventuellement vide de segments décroissants, de cardinal $t_{\rho, \tau^{\prime}} \geq 0$ inférieur ou égal à $\left[\left(a_{\rho, \tau_{0}}\right) / 2\right]+1$, de la forme :

$$
\left\{\left[x_{i},\left(a_{\rho, \tau_{0}}+1\right) / 2-i+1\right] ; i \in\left[1, t_{\rho, \tau^{\prime}}\right]\right\},
$$

où $x_{1}<\cdots<x_{t_{\rho, \tau^{\prime}}}$, avec une inclusion :

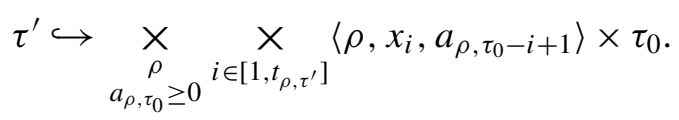

La démonstration est sans difficulté et on renvoie à loc.cit. pour les détails. On a alors :

$$
\pi_{\tau^{\prime}}=\underset{\substack{\rho \\ a_{\rho, \tau_{0}}>0}}{\times}\left(\underset{i \in\left[1, t_{\rho, \tau^{\prime}}\right]}{\times} \operatorname{St}\left(\rho, 2 x_{i}+1\right) \underset{a<a_{\rho, \tau_{0}}-2 t_{\rho, \tau^{\prime}}}{\times} \operatorname{St}(\rho, a)\right) \underset{\substack{\rho \\ a_{\rho, \tau_{0}}=0 \\ t_{\rho, \tau^{\prime}}=1}}{\times} \operatorname{St}\left(\rho, 2 x_{1}\right) .
$$


Il faut remarquer que si $a_{\rho, \tau_{0}}=0$ alors $t_{\rho, \tau^{\prime}}$ vaut soit 0 soit 1 . Cela termine la preuve.

Proposition. Soit $\tau$ une représentation elliptique de $U(n, E / F)$ et soit $\tilde{\pi}$ une représentation elliptique irréductible de $\tilde{G}_{n}$. On suppose que $\tilde{\pi}$ dans la décomposition 1.4 fournit un élément de $I_{\text {cusp }}^{\langle U(n) \text {,st }}(U(n))$ non orthogonal à $\tau$. Alors $\tau$ est une série discrète et $\tilde{\pi}$ est un prolongement de $\pi_{\tau}$. En particulier $\pi_{\tau}$ est $\theta$-discrète.

On écrit $\tilde{\pi}$ comme un prolongement de la représentation :

$$
\pi \simeq \underset{(\rho, a) \in \mathscr{E}}{\times} \operatorname{St}(\rho, a)
$$

où $\mathscr{E}$ est un ensemble de couples formés d'une représentation cuspidale irréductible $\theta$ invariante $\rho$ et d'un entier $a$; cet ensemble est sans multiplicité. On considère la représentation

$$
\pi^{+}: \simeq \underset{(\rho, a) \in \mathscr{E}}{\times} \operatorname{St}(\rho, a+2) .
$$

C'est une représentation de $\operatorname{GL}\left(n^{+}, E\right)$ où $n^{+}=n+2 \sum_{(\rho, a) \in \mathscr{E}} d_{\rho}$. On ordonne $\mathscr{E}$ de telle sorte que si $\left(\rho^{\prime}, a^{\prime}\right)$ précède $\left(\rho^{\prime \prime}, a^{\prime \prime}\right)$ alors que $\rho^{\prime} \simeq \rho^{\prime \prime}$ alors $a^{\prime}<a^{\prime \prime}$. On calcule les modules de Jacquet successifs

$$
\operatorname{Jac}_{\rho \|\left.\right|_{E} ^{(a+1) / 2}}^{\theta} \cdots \operatorname{Jac}_{\rho^{\prime} \mid \|_{E}^{\left(a^{\prime}+1\right) / 2}}^{\theta} \tilde{\pi}^{+}
$$

où l'ordre écrit ci-dessus et l'ordre inverse de celui de $\mathscr{E} ;$ c'est-à-dire que l'on commence par le premier élément pour aller vers le dernier. Chaque opération donne une représentation elliptique de la forme :

$$
\underset{(\rho, a) \in \mathscr{C}_{\leq}}{\times} \operatorname{St}(\rho, a) \underset{(\rho, a) \in \mathscr{E}_{>}}{\times} \operatorname{St}(\rho, a+2),
$$

où $\mathscr{E}_{\leq}$est le sous-ensemble de $\mathscr{E}$ utilisé pour prendre les modules de Jacquet et $\mathscr{E}_{>}$celui qui n'a pas encore été utilisé. A la dernière étape, on trouve $\tilde{\pi}$. On vérifie alors qu'il existe $\tau_{+}$intervenant dans $I_{\text {cusp }}^{\left\langle U\left(n^{+}\right) \text {,st }\right\rangle}\left(U\left(n^{+}, E / F\right)\right.$ et tel que $\tau$ soit un sous-quotient de $\mathrm{Jac}_{\rho \|_{E}^{(a+1) / 2} ;(\rho, a) \in \mathscr{E}} \tau^{+}$où là aussi les $(\rho, a)$ sont pris dans l'ordre indiqué ci-dessus. Le point ici est d'utiliser (1-3) et de montrer que les termes que l'on obtient sur $\langle U(n)$, st $\rangle$ ne peuvent provenir que de la donnée endoscopique $\left\langle U\left(n^{+}\right)\right.$, st $\rangle$et non d'une donnée de la forme $\left\langle U\left(n_{1}\right) \times U\left(n_{2}\right)\right\rangle$ avec $n_{2}>0$. Ceci est juste une propriété du support cuspidal ordinaire; en effet s'il n'en était pas ainsi, il existerait une représentation elliptique de $U\left(n_{2}, E / F\right)$, notée $\tau^{\prime}$, dont le support cuspidal ordinaire serait un sous-ensemble de $\left\{\rho||_{E}^{(a+1) / 2} ;(\rho, a) \in \mathscr{E}\right\}$, en particulier ce support cuspidal n'est formé que de représentations de groupes linéaires. Les exposants sont en valeur absolue, supérieurs ou égaux à 1 . Avec 4.1 on vérifie que $\tau^{\prime}$ est une série discrète et pas seulement une représentation 
elliptique. Son support cuspidal étendu est alors par définition inclus dans

$$
\left\{\rho||^{(a+1) / 2},\left.\rho\right|^{-(a+1) / 2} ;(\rho, a) \in \mathscr{E}\right\} ;
$$

mais un tel support n'est pas une union de segments centrés en 0 et ne peut donc être le support cuspidal d'une représentation tempérée d'un groupe linéaire. Ceci contredirait le lemme ci-dessus.

On a donc prouvé l'existence de $\tau^{+}$. Par construction le support cuspidal ordinaire de $\tau^{+}$se déduit de celui de $\tau$ en ajoutant l'ensemble $\left\{\rho||_{E}^{(a+1) / 2} ;(\rho, a) \in \mathscr{E}\right\}$ d'où

$$
\operatorname{Supp} \operatorname{cusp}\left(\tau^{+}\right)=\operatorname{Supp} \operatorname{cusp}(\tau) \bigcup_{(\rho, a) \in \mathscr{E}}\left\{\left(\rho||^{(a+1) / 2}, \rho||^{-(a+1) / 2}\right\} .\right.
$$

Puisque $\tau^{+}$est une représentation elliptique, on sait aussi que son support cuspidal étendu s'écrit sous la forme :

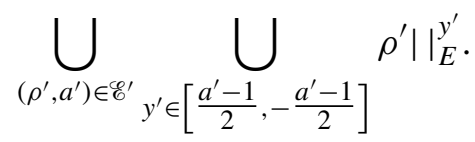

On revient à 4.1 pour savoir simplement que dans l'inclusion de cette référence, les segments de $\mathscr{E}$ (avec les notations de loc. cite) sont nécessairement de la forme $\left\langle\rho^{\prime},\left(a^{\prime}-1\right) / 2, b^{\prime}\right\rangle$ avec l'existence de $\left(\rho^{\prime}, a^{\prime}\right) \in \mathscr{E}^{\prime}$ et soit $b^{\prime}>0$ soit $b^{\prime}$ est lui-même de la forme $-\left(a^{\prime \prime}-1\right) / 2$ avec $\left(\rho^{\prime}, a^{\prime \prime}\right) \in \mathscr{E}$ (voir la preuve du lemme ci-dessus).

Ainsi quand on applique les formules standard de Bernstein-Zelevinski pour calculer les modules de Jacquet, on a $\mathrm{Jac}_{\rho^{\prime}||_{E}^{x}} \tau^{+}=0$ pour $x>0$ sauf s'il existe $\left(\rho^{\prime}, a^{\prime}\right) \in \mathscr{E}^{\prime}$ avec $x=\left(a^{\prime}-1\right) / 2$. De plus pour un tel terme la filtration correspondante est réduite à un élément : il faut changer le segment qui est soit de la forme $\left\langle\rho^{\prime},\left(a^{\prime}-1\right) / 2, b^{\prime}\right\rangle$ et $\left\langle\rho^{\prime},\left(a^{\prime}-1\right) / 2-1, b^{\prime}\right\rangle$ soit de la forme $\left\langle\rho^{\prime}, A^{\prime},-\left(a^{\prime}-1\right) / 2\right\rangle$ en $\left\langle\rho^{\prime}, A^{\prime},-\left(a^{\prime}-1\right) / 2+1\right\rangle$. Ainsi ce terme n'intervient plus pour les autres modules de Jacquet à cause de l'ordre mis. Et on montre progressivement que la non nullité du module de Jacquet de $\tau^{+}$entraîne que pour chaque $(\rho, a) \in \mathscr{E}$, il existe $\left(\rho^{\prime}, a^{\prime}\right) \in \mathscr{E}^{\prime}$ avec $\rho^{\prime} \simeq \rho$ et $a^{\prime}=a+2$.

En d'autres termes pour tout $(\rho, a) \in \mathscr{E}$, il existe $(\rho, a+2) \in \mathscr{E}^{\prime}$; on note $\mathscr{E}^{\prime \prime}$ l'ensemble restant. Quand on passe de $\operatorname{Supp} \operatorname{cusp}\left(\tau^{+}\right)$à $\operatorname{Supp} \operatorname{cusp}(\tau)$ on enlève précisément les extrêmités des segments non dans $\mathscr{E}^{\prime \prime}$ et on ne touche pas aux éléments de $\mathscr{E} \prime \prime$; les couples non dans $\mathscr{E}^{\prime \prime}$ fournissent donc au support cuspidal étendu de $\tau$ exactement le support cuspidal de $\pi$ et il ne reste donc plus qu'à démontrer que $\mathscr{E} "$ " est vide. Mais, on a, puisque $\mathscr{E}$ donne le support cuspidal de $\pi$

$$
n=\sum_{(\rho, a) \in \mathscr{E}} a d_{\rho} .
$$


D'où $n^{+}=\sum_{(\rho, a) \in_{\mathscr{E}}^{\mathscr{E}}}(a+2) d_{\rho}=\sum_{\left(\rho^{\prime}, a^{\prime}\right) \in_{\mathscr{E}^{\prime}-\mathscr{E}^{\prime \prime}}} a^{\prime} d_{\rho^{\prime}}$. Et par définition du support cuspidal étendu de $\tau^{+}$

$$
n^{+}=\sum_{\left(\rho^{\prime}, a^{\prime}\right) \in \mathscr{E}^{\prime}} a^{\prime} d_{\rho^{\prime}}
$$

D'où $\mathscr{E}^{\prime \prime}=\varnothing$; ceci a plusieurs conséquences. On sait que le support cuspidal étendu de $\tau$ correspond à un ensemble de segments centrés à l'origine, ensemble sans multiplicité. Ainsi $\tau$ est une série discrète et pas seulement une représentation elliptique. La fin de la preuve est alors claire.

\subsection{Première description des paquets stables de séries discrètes pour les groupes}

unitaires. Soit $\tau$ une série discrète irréductible de $U(n, E / F)$; on a défini le support cuspidal étendu de $\tau$ que l'on peut voir comme le support cuspidal de la représentation $\pi_{\tau}$ déjà définie. Réciproquement soit $\pi$ une représentation $\theta$-discrète de $\operatorname{GL}(n, E)$, on note $\Pi(\pi)$ l'ensemble des séries discrètes de $U(n, E / F)$ ayant comme support cuspidal éténdu le support cuspidal de $\pi$.

Proposition. Soit $\pi$ une série $\theta$-discrète de $\mathrm{GL}(n, E)$; on suppose que $\Pi(\pi)$ est non vide. Il existe une unique (à un scalaire près) combinaison linéaire stable de séries discrètes irréductibles de $U(n, E / F)$ de la forme:

$$
\sum_{c_{\tau}, \tau \in \Pi(\pi)} c_{\tau} \tau
$$

De plus pour tout $\tau \in \Pi(\pi), c_{\tau} \neq 0$.

Soit $\tau \in \Pi(\pi)$; on sait que la projection, $f_{\tau}^{\text {st }}$ de $\tau$ sur $I_{\text {cusp }}^{\text {st }}(U(n, E / F))$ est non nulle et il existe donc une combinaison linéaire d'éléments, $f$, de $I_{\text {cusp }}\left(\tilde{G}_{n}\right)$ qui correspond à $f_{\tau}^{\text {st }}$ dans 1.4 . Il y a donc au moins une représentation elliptique $\tilde{\pi}$ intervenant dans $f$ telle que sa décomposition fasse intervenir $\tau$. On a vu que $\tilde{\pi}$ est un prolongement de $\pi_{\tau}=\pi$. Ainsi la projection de $\pi_{\tau}$ sur $I_{\text {cusp }}^{\text {st }}\left(\tilde{G}_{n}\right)$ donne une distribution stable non nulle de $U(n, E / F)$. On sait que toutes les séries discrètes de $U(n, E / F)$ intervenant dans cette décomposition ont comme support cuspidal étendu celui de $\pi_{\tau}$, c'est-à-dire sont dans $\Pi(\pi)$. D'où l'existence d'une distribution stable ayant les propriétés de l'énoncé ; cette distribution vérifie $c_{\tau} \neq 0$ pour le $\tau$ que nous avons fixé. Montrons maintenant l'unicité annoncée dans l'énoncé. Soit donc $f^{\text {st }}, f^{\prime}$,st $\in I_{\text {cusp }}^{\text {st }}\left(U(n, E / F)\right.$ dont le support est inclus dans $\Pi(\pi)$. On note $f, f^{\prime}$ les images de ces éléments dans $I_{\text {cusp }}\left(\tilde{G}_{n}\right)$. En utilisant d'abord une représentation $\tau$ dans le support de $f^{\text {st }}$ puis une représentation $\tau^{\prime}$ dans le support de $f^{\prime}{ }^{\prime} t$, on vérifie comme ci-dessus qu'un prolongement de $\pi$ intervient dans $f$ et dans $f^{\prime}$ avec un coefficient non nul ; changer de prolongement revient à changer les signes dans $f^{\text {st }}$ ou $f^{\prime}$ st et on peut donc supposer que c'est le même prolongement, $\tilde{\pi}$, qui intervient. Quitte à multiplier $f^{\text {st }}$ par un nombre complexe, on peut supposer que 
$f-f^{\prime}$ n'a plus $\tilde{\pi}$ dans son support. L'image de $f-f^{\prime}$ dans 1.4 est précisément $f^{\text {st }}-f^{\prime s t}$. Si $f^{\text {st }}-f^{\prime}$ st était non nul, il faudrait que $f-f^{\prime}$ ait un coefficient non nul sur $\tilde{\pi}$ ce qui est exclu. D'où $f^{\text {st }}=f^{\prime s t}$ comme annonçé.

Comme pour chaque élément de $\Pi(\pi)$ il existe $f^{\text {st }}$ satisfaisant les hypothèses de l'énoncé avec $c_{\tau} \neq 0$, l'unicité que l'on vient de démontrer assure la propriété de non nullité, $c_{\tau} \neq 0$ pour tout $\tau \in \Pi(\pi)$. Cela termine la preuve.

5.6. Support cuspidal et pôle de fonctions $L$. Soit $\rho$ une représentation cuspidale irréductible, $\theta$-invariante de $\operatorname{GL}\left(d_{\rho}, E\right)$. On note $L\left(\rho, r_{A}^{\prime}, s\right)$ la fonction $L$ d'Asai-Shahidi de $\rho$; cette fonction $L$ s'introduit naturellement grâce aux travaux de Shahidi [1990] complétés par ceux de Goldberg [1994], c'est elle qui contrôle l'irréductibilité de l'induite de $\rho$ au groupe unitaire quasidéployé $U\left(2 d_{\rho}, E / F\right)$ à partir du parabolique de Levi $\operatorname{GL}\left(d_{\rho}, E\right)$. Cette induite est irréductible si et seulement si $L\left(\rho, r_{A}^{\prime}, s\right)$ a un pôle en $s=0$. Si cette induite est irréductible alors l'induite dans la même situation de $\rho||_{E}^{1 / 2}$ est, elle, réductible. Et vice et versa.

Proposition. Soit $\tau$ une série discrète irréductible de $U(n, E / F)$ et $\rho$ comme cidessus; on suppose qu'il existe un entier a tel que $\rho||_{E}^{(a-1) / 2}$ est dans le support cuspidal étendu de $\tau$. Alors a est pair si $L\left(\rho, r_{A}^{\prime}, s\right)$ a un pôle en $s=0$ et impair sinon.

Une autre façon de dire les choses peut-être plus parlante est la suivante : on a défini $\pi_{\tau}$ comme représentation $\theta$-discrète de $\operatorname{GL}(n, E)$ telle que son support cuspidal est exactement le support cuspidal étendu de $\tau$. On écrit

$$
\pi_{\tau}=\underset{(\rho, a) \in \mathscr{E}}{\times} \operatorname{St}(\rho, a) .
$$

Et la proposition dit que si $(\rho, a) \in \mathscr{E}$ alors $a$ est pair si et seulement si $L\left(\rho, r_{A}^{\prime}, s\right)$ a un pôle en $s=0$.

On suppose d'abord que $\tau$ est cuspidal; on note $x_{0}$ l'unique réel positif ou nul tel que $\rho||^{x_{0}} \times \tau$ soit réductible. On sait déjà que $2 x_{0}+1$ est un entier et on va montrer qu'il est impair si et seulement si $L\left(\rho, r_{A}^{\prime}, s\right)$ n'a pas de pôle en $s=0$. Cela suffit grâce à [Mœglin 2002] appendice que l'on ne récrit pas ici. On fait remarquer au lecteur que l'on démontre ici même un peu plus que ce qui est annoncé dans l'énoncé puisque l'on caractérise tous les points de réductibilité des induites de cuspidales.

Le caractère $\omega$ non trivial de $E^{*}$, dont la restriction à $F^{*}$ est le caractère de $F^{*}$ correspondant par la théorie du corps de classe à l'extension $E$ de $F$ est celui qui $s$ 'introduit dans les choix pour l'endoscopie.

Supposons d'abord que $L\left(\rho, r_{A}^{\prime}, s\right)$ n'a pas de pôle en $s=0$. En suivant [Goldberg 1994, introduction, (1)] on sait que c'est alors $L\left(\omega \otimes \rho, r_{A}^{\prime}, s\right)$ qui a un 
pôle en $s=0$. Ainsi l'induite pour $U\left(2 d_{\rho}, E / F\right)$ de la représentation $\omega \otimes \rho||_{E}^{1 / 2}$ contient une sous-représentation irréductible, $\tau_{2}$ qui est une série discrète. On note $f_{\tau}^{\text {st }}$ la projection de $\tau$ sur $I_{\text {cusp }}^{\text {st }}(U(n, E / F))$ et $f_{2}^{\text {st }}$ la projection de $\tau_{2}$ sur $I_{\text {cusp }}^{\text {st }}\left(U\left(2 d_{\rho}, E / F\right)\right)$. On considère l'élément $f_{\tau}^{\text {st }} \otimes f_{2}^{\text {st }}$ de

$$
I_{\text {cusp }}^{\left\langle U(n) \times U\left(2 d_{\rho}\right)\right\rangle}\left(U(n, E / F) \times U\left(2 d_{\rho}, E / F\right)\right)
$$

qu'il définit et l'image réciproque $\mathscr{F}$ dans $I_{\text {cusp }}\left(\tilde{G}_{n+2 d_{\rho}}\right)$ de cet élément (voir 1.4). On rappelle que l'on identifie un élément de $I_{\text {cusp }}^{\langle H\rangle}$ et une représentation virtuelle de $H$ et on utilise la notation pour les modules de Jacquet donnée à la fin de 1.6.

On applique (1-3) à ces éléments pour $\sigma=\rho||_{E}^{1 / 2}$. On veut vérifier que le terme de droite de cette équation contient l'élément $f_{\tau}^{\text {st }}$ comme composante sur $I_{\text {cusp }}^{\langle U(n), \text { st }\rangle}(U(n, E / F))$; pour cela, il suffit de vérifier que $f_{2}^{\text {st }}$ contient $\tau_{2}$ et des représentations $\tau^{\prime}$ telles que $\mathrm{Jac}_{\omega \rho||_{E}^{1 / 2}} \tau^{\prime}=0$. Soit $\tau^{\prime}$ une représentation irréductible de $U\left(2 d_{\rho}, E / F\right)$ dont le module de Jacquet contient comme sous-quotient la représentation $\omega \rho||_{E}^{1 / 2}$ du sous-groupe de Levi $\operatorname{GL}\left(d_{\rho}, E\right)$; un tel $\tau^{\prime}$ est certainement un sous-quotient de l'induite de $\omega \rho||_{E}^{1 / 2}$. Mais le seul sous-quotient de cette induite qui soit tempérée est $\tau_{2}$ d'où l'assertion cherchée. Ainsi

$$
\operatorname{Jac}_{\rho \|_{E}^{\theta / 2}}^{1 / 2}
$$

est non nul avec les notations de loc.cite. Il est immédiat que $\mathrm{Jac}_{\rho \|_{E}}^{\theta} \mathscr{F}_{E}^{1 / 2}$ est encore à support dans les représentations $\theta$-discrètes (on enlève un facteur $\operatorname{St}(\rho, 2)$ quand un tel facteur apparaît et sinon on trouve 0 ). Et la projection de cet élément sur $I_{\text {cusp }}^{\text {st }}(U(n, E / F))$ doit donc coïncider avec $f_{\tau}^{\text {st }}$. Cela veut dire que $\mathscr{F}$ a une composante non nulle sur $\pi_{\tau} \times \operatorname{St}(\rho, 2)$. On sait au départ que $\pi_{\tau} \times \operatorname{St}(\rho, 2)$ est $\theta$-discrète, cela veut dire que si l'on écrit :

$$
\pi_{\tau}=\underset{\left(\rho^{\prime}, a^{\prime}\right) \in \mathscr{E}}{\times} \operatorname{St}\left(\rho^{\prime}, a^{\prime}\right)
$$

l'ensemble $\mathscr{E}$ ne contient pas $(\rho, 2)$. Or on sait que si $a_{\rho, \tau} \geq 1$ alors d'une part $x_{\rho, \tau}=\left(a_{\rho, \tau}+1\right) / 2$ et d'autre part si $a_{\rho, \tau}$ est pair, alors $\mathscr{E}$ contient $(\rho, 2)$. On a donc montré que si $a_{\rho, \tau} \geq 1$ c'est un entier impair et $2 x_{\rho, \tau}+1$ est un entier pair. On veut aussi montrer que le cas où $x_{\rho, \tau}=1 / 2$ est impossible sous nos hypothèses. Il faut faire le même raisonnement mais en remplaçant $\tau$ par l'unique sous-module irréductible de l'induite $\tau^{\prime}:=\rho||_{E}^{1 / 2} \times \tau$. La cuspidalité de $\tau$ n'a servi à rien cidessus. Avec les hypothèses, $\pi_{\tau^{\prime}}$ est de la forme $\operatorname{St}(\rho, 2) \times \pi^{\prime}$ avec $\pi^{\prime}$ convenable et on obtient la contradiction cherchée.

Réciproquement supposons que $\left(a_{\rho, \tau}+1\right) / 2$ est entier non demi-entier, c'està-dire que soit $a_{\rho, \tau}=-1$ soit $a_{\rho, \tau}$ est un entier impair. On a défini, $\pi_{\tau}$ et on 
considère

$$
\pi:=\operatorname{St}(\rho, 2) \times \pi_{\tau} .
$$

C'est encore une représentation $\theta$ discrète. On en fixe un prolongement $\tilde{\pi}$ à $G_{n+2 d_{\rho}}^{+}$. On regarde la décomposition de l'image de $\tilde{\pi}$ dans $I_{\text {cusp }}\left(\tilde{G}_{n+2 d_{\rho}}\right)$ suivant 1.4. On note $f^{\langle H\rangle}$ les différentes composantes quand $\langle H\rangle$ parcourt l'ensemble des groupes endoscopiques elliptiques de $\tilde{G}_{n+2 d_{\rho}}$. On calcule le module de Jacquet $\operatorname{Jac}_{\rho \mid \perp^{1 / 2}}^{\theta} \pi$ et on obtient $\pi_{\tau}$. Cela permet de retrouver la décomposition de $\pi_{\tau}$ dans $I_{\text {cusp }}(\tilde{G})$ en utilisant (1-3); on ne s'interesse qu'à la composante suivant $I_{\text {cusp }}^{\langle U(n), \text { st }\rangle}(U(n, E / F)$. Il faut donc considérer

$$
\operatorname{Jac}_{\rho \|_{E}^{1 / 2}} f^{\left\langle U\left(n+2 d_{\rho}\right), \text { st }\right\rangle}
$$

(avec les notations de la même équation) et $\mathrm{Jac}_{\omega \rho||_{E}^{1 / 2}} f^{\left\langle U(n) \times U\left(2 d_{\rho}\right)\right\rangle}$ où le module de Jacquet porte sur les représentations de $U\left(2 d_{\rho}, E / F\right)$. Ce terme est facile à calculer : il vaut 0 sauf si la représentation $\tau_{2}$ construite ci-dessus existe, c'est-àdire si $L\left(\rho, r_{A}^{\prime}, s\right)$ n'a pas de pôle en $s=0$. Sous cette hypothèse, on écrit

$$
f^{\left\langle U(n) \times U\left(2 d_{\rho}\right)\right\rangle}=f_{1} \otimes \tau_{2} \oplus f^{\prime},
$$

où $f^{\prime}$ ne fait plus intervenir $\tau_{2}$ et

$$
\operatorname{Jac}_{\omega \rho||_{E}^{1 / 2}} f^{\left\langle U(n) \times U\left(2 d_{\rho}\right)\right\rangle}=f_{1} .
$$

Si $L\left(\rho, r_{A}^{\prime}, s\right)$ n'a pas de pôle en $s=0$, on a la réciproque cherchée. On raisonne donc par l'absurde en supposant que cette fonction $L$ a un pôle. Alors, nécessairement $\mathrm{Jac}_{\rho||^{1 / 2}} f^{\left\langle U\left(n+2 d_{\rho}\right) \text {,st }\right\rangle}$ est la projection de $\pi_{\tau} \operatorname{sur} U^{\text {st }}(U(n, E / F))$. Cette projection contient $\tau$ et il existe donc une représentation dans le support de $f^{\left\langle U\left(n+2 d_{\rho}\right) \text {,st }\right\rangle}$ qui admet $\rho||^{1 / 2} \otimes \tau$ comme sous-quotient dans son module de Jacquet. Cette représentation est une série discrète et l'induite $\rho||^{1 / 2} \times \tau$ doit donc être réductible. Ceci est contradictoire avec le fait que $x_{\rho, \tau}$ est entier non demientier. Cela termine la preuve.

5.7. Paquets stables de séries discrètes pour les groupes unitaires. Soit $\psi$ un homorphisme de $W_{E} \times \operatorname{SL}(2, \mathbb{C})$ dans $\operatorname{GL}(n, \mathbb{C})$. On suppose que $\psi$ est semi-simple borné sur $W_{E}$ et continu au sens usuel. On suppose que la représentation $\psi$ est sans multiplicité et qu'elle se décompose en somme de représentations irréductibles,

$$
\psi=\bigoplus_{(\rho, a) \in \mathscr{E}} \rho \otimes \sigma_{a},
$$

où ici $\rho$ est une représentation irréductible de dimension finie $d_{\rho}$ de $W_{E}$ et $a$ un entier, $\sigma_{a}$ étant l'unique représentation irréductible de $\operatorname{SL}(2, \mathbb{C})$ de dimension $a$. On dit que $\psi$ est $\theta$-discrète si $\psi$ est sans multiplicité et si toutes les représentations $\rho$ intervenant dans (5-4) sont invariantes sous l'action du composé de $g \mapsto^{t} g^{-1}$ et de la conjugaison dans $W_{E}$ venant de l'extension $E / F$. 
On dit que $\psi$ est stable si dans sa décomposition (5-4), on a en plus que pour tout $(\rho, a) \in \mathscr{E}, a$ est pair si et seulement si $L\left(\rho, r_{A}^{\prime}, s\right)$ a un pôle en $s=0$; ici il est plus simple de dire que dans cette fonction $L, \rho$ est la représentation cuspidale de $\operatorname{GL}\left(d_{\rho}, E\right)$ correspondant à $\rho$ par la correspondance de Langlands. Bien sûr on peut interpréter cette définition par le fait que $\psi$ se prolonge en un homomorphisme de $W_{F} \times \operatorname{SL}(2, \mathbb{C})$ dans le groupe dual de $U(n, E / F)$. Mais on reste ici beaucoup plus élémentaire.

On rappelle encore que la correspondance de Langlands associe à tout morphisme $\psi \theta$-discret une représentation $\theta$-discrète de $\mathrm{GL}(n, E)$ que l'on note $\pi(\psi)$. On sait associer à $\pi(\psi)$ un paquet stable de séries discrètes de $U(n, E / F)$ (qui éventuellement peut être vide) en prenant la projection de l'image d'un prolongement de $\pi(\psi)$ dans $I_{\text {cusp }}\left(\tilde{G}_{n}\right)$ sur $I_{\text {cusp }}^{\langle U(n), \text { st }\rangle}(U(n, E / F))$. On note $\Pi(\psi)$ le paquet obtenu. On a montré en 5.5 que $\Pi(\psi)$ est exactement formé des séries discrètes de $U(n, E / F)$ dont le support cuspidal étendu coïncide avec le support cuspidal de $\pi(\psi)$. Il ne nous reste plus qu'à compléter cette description en montrant que $\Pi(\psi)$ est non vide exactement quand $\psi$ est stable. C'est l'objet du théorème suivant :

Théorème. Il existe une bijection entre l'ensemble des paquets stables de séries discrètes de $U(n, E / F)$ et l'ensemble des classes de conjugaison de morphismes $\theta$-discrets et stables de $W_{E} \times \mathrm{SL}(2, \mathbb{C})$ dans $\operatorname{GL}(n, \mathbb{C})$. La bijection est celle expliquée ci-dessus.

Le théorème résulte exactement des propositions en 5.5 et 5.6.

\section{Changement de base}

Le but de cette partie est de démontrer qu'à toute représentation $\theta$-discrète $\pi$ de $\operatorname{GL}(n, E)$ est associée une unique donnée endoscopique elliptique $\langle H\rangle$ de $\tilde{G}_{n}$ telle que l'image d'un prolongement de $\pi$ dans $I_{\text {cusp }}\left(\tilde{G}_{n}\right)$ soit exactement dans l'image de $I_{\text {cusp }}^{\langle H\rangle}(H)$ dans la décomposition 1.4. On va démontrer cela, en montrant que $\langle H\rangle$ est uniquement déterminé par le support cuspidal de $\pi$.

Soit $n=n_{1}+n_{2}$ une décomposition de $n$, le couple $\left(n_{1}, n_{2}\right)$ est ordonné. On en déduit une donnée endoscopique $\langle H\rangle$ de $\tilde{G}_{n}$.

Soient $\tau_{1}$ et $\tau_{2}$ des séries discrètes de $U\left(n_{1}, E / F\right)$ et de $U\left(n_{2}, E / F\right)$; on les suppose irréductibles. On leur a associé via le support cuspidal des représentations $\pi_{\tau_{1}}$ et $\pi_{\tau_{2}}$ de $\operatorname{GL}\left(n_{1}, E\right)$ et $\operatorname{GL}\left(n_{2}, E\right)$ respectivement. On pose :

$$
\pi_{\tau_{1} \otimes \tau_{2}}=\pi_{\tau_{1}} \times\left(\omega \otimes \pi_{\tau_{2}}\right),
$$

où $\omega$ est comme ci-dessus un caractère de $E^{*}$ dont la restriction à $F^{*}$ est le caractère de $F^{*}$ correspondant à l'extension $E / F$.

Lemme. Soit $\pi$ une représentation $\theta$-discrète de $\mathrm{GL}(n, E)$ dont on fixe un prolongement $\tilde{\pi}$ à $\tilde{G}_{n}$. On note $f_{\pi}$ l'image de $\tilde{\pi}$ dans $I_{\text {cusp }}\left(\tilde{G}_{n}\right)$. On suppose que la 
projection de $f_{\pi}$ sur $I_{\mathrm{cusp}}^{\langle H\rangle}(H)$ est non nulle. Alors $\pi$ est de la forme $\pi_{\tau_{1} \otimes \tau_{2}}$ pour $\tau_{1}$ et $\tau_{2}$ convenables.

Ce lemme, dans le cas où $n_{1}=n$, est un cas particulier de la proposition de 5.4 ; il s'en déduit aussi si $n_{2}=n$, puisque la tensorisation par $\omega$ ramène au cas stable. La démonstration du cas général suit le même principe. On fixe $\pi$ comme dans l'énoncé que l'on écrit :

$$
\pi=\underset{(\rho, a) \in \mathscr{E}}{\times} \operatorname{St}(\rho, a) .
$$

On note $\pi^{+}:=\times_{(\rho, a) \in \mathscr{E}} \operatorname{St}(\rho, a+2) ;$ c'est une représentation de $\operatorname{GL}\left(n^{+}, E\right)$ où $n^{+}$est convenable. Et on ordonne encore $\mathscr{E}$ de façon à ce que si $\left(\rho^{\prime}, a^{\prime}\right)$ précède $\left(\rho^{\prime \prime}, a^{\prime \prime}\right)$ avec $\rho^{\prime}=\rho^{\prime \prime}$ alors $a^{\prime}<a^{\prime \prime}$. On fixe un prolongement $\tilde{\pi}^{+}$de $\pi^{+}$et on regarde l'image de $\tilde{\pi}^{+}$dans $I_{\text {cusp }}\left(\tilde{G}_{n^{+}}\right)$et la décomposition de cet élément suivant 1.4. On va encore prendre les modules de Jacquet successifs suivant les éléments $\rho^{\prime}||_{E}^{\left(a^{\prime}+1\right) / 2}$ pour $\left(\rho^{\prime}, a^{\prime}\right)$ parcourant $\mathscr{E}$ dans l'ordre. A chaque étape on obtient une représentation elliptique et à la fin on trouve $\pi$. Quand on applique successivement (1-3), on obtient simplement l'existence d'une décomposition de $\mathscr{E}$ en deux sousensembles $\mathscr{E}_{1} \cup \mathscr{E}_{2}$, une donnée endoscopique $\left\langle U\left(n_{1}^{+}\right) \times U\left(n_{2}^{+}\right)\right\rangle$de $\tilde{G}_{n^{+}}$et un élément $\tau_{1}^{+} \otimes \tau_{2}^{+}$dans le support de la projection de $\tilde{\pi}^{+}$sur

$$
I_{\text {cusp }}^{\left\langle U\left(n_{1}^{+}\right) \times U\left(n_{2}^{+}\right)\right\rangle}\left(U\left(n_{1}^{+}, E / F\right) \times U\left(n_{2}^{+}, E / F\right)\right)
$$

tel que $\tau_{i}^{+}, i=1,2$ ait dans son module de Jacquet un sous-quotient isomorphe à

$$
\bigotimes_{\left(\rho^{\prime}, a^{\prime}\right) \in \mathscr{E}_{i}} \rho^{\prime}||_{E}^{\left(a^{\prime}+1\right) / 2} \otimes \tau_{i}
$$

On sait que $\tau_{1}$ est une série discrète; en particulier si $\left(\rho^{\prime}, a^{\prime}\right) \in \mathscr{E}_{1}$ alors $a^{\prime}$ est pair exactement si $L\left(\rho^{\prime}, r_{A}^{\prime}, s\right)$ a un pôle en $s=0$. De même si $\left(\rho^{\prime}, a^{\prime}\right) \in \mathscr{E}_{2}$ alors $a^{\prime}$ est pair exactement si $L\left(\omega \rho^{\prime}, r_{A}^{\prime}, s\right)$ a un pôle en $s=0$; c'est la condition opposée à la précédente puisqu'elle est équivalente à $L\left(\rho^{\prime}, r_{A}^{\prime}, s\right)$ n'a pas de pôle en $s=0$. Ainsi la décomposition de $\mathscr{E}$ en $\mathscr{E}_{1} \cup \mathscr{E}_{2}$ est complètement déterminée a priori. Ensuite ce sont les arguments standard :

$$
\operatorname{Supp} \operatorname{cusp}\left(\tau_{1}^{+}\right)=\bigcup_{\left(\rho^{\prime}, a^{\prime}\right) \in \mathscr{E}_{1}}\left\{\rho^{\prime}||^{\left(a^{\prime}+1\right) / 2}, \rho^{\prime}||^{-\left(a^{\prime}+1\right) / 2}\right\} \cup \operatorname{Supp} \operatorname{cusp}\left(\tau_{1}\right) .
$$

Cela prouve encore que

$$
\pi_{\tau_{1}}=\underset{\left(\rho^{\prime}, a^{\prime}\right) \in \mathscr{E}_{1}}{\times} \operatorname{St}\left(\rho^{\prime}, a^{\prime}\right) \times \pi_{1}^{\prime},
$$

où $\pi_{1}^{\prime}$ est une représentation $\theta$-discrète convenable. On fait la même chose en remplaçant 1 par 2 et on trouve que 


$$
\pi_{\tau_{2}}=\underset{\left(\rho^{\prime}, a^{\prime}\right) \in \mathscr{C}_{2}}{\times} \omega \otimes \operatorname{St}\left(\rho^{\prime}, a^{\prime}\right) \times \pi_{2}^{\prime}
$$

où $\pi_{2}^{\prime}$ est une représentation $\theta$-discrète convenable. En comparant les dimensions, on trouve encore que $\pi_{1}^{\prime}$ et $\pi_{2}^{\prime}$ sont nécessairement triviales. En mettant ensemble (6-1) et (6-2) tordu par $\omega$, on obtient

$$
\pi_{\tau_{1} \otimes \tau_{2}}=\pi_{\tau_{1}} \times\left(\omega \otimes \pi_{\tau_{2}}\right)=\underset{\left(\rho^{\prime}, a^{\prime}\right) \in \mathscr{C}}{\times} \operatorname{St}\left(\rho^{\prime}, a^{\prime}\right)=\pi .
$$

D'où le lemme.

\subsection{Changement de base réciproque.}

Théorème. Soit $\pi$ une représentation $\theta$ discrète de $\mathrm{GL}(n, E)$. Alors il existe une donnée endoscopique elliptique $\langle H\rangle$ de $\tilde{G}_{n}$ tel que $\pi$ soit $\langle H\rangle$-stable. Et $\langle H\rangle$ est évidemment unique avec cette propriété.

Soit $\pi$ comme dans l'énoncé et $\tilde{\pi}$ un prolongement de $\pi$; on note $f_{\pi}$ l'image de $\pi$ dans $I_{\text {cusp }}\left(\tilde{G}_{n}\right)$. Soit $\langle H\rangle$ une donnée endoscopique de $\tilde{G}_{n}$ telle que $f_{\pi}$ ait une projection non nulle sur $I_{\text {cusp }}^{\langle H\rangle}(H)$. On écrit $\langle H\rangle=\left\langle U\left(n_{1} \times U\left(n_{2}\right)\right\rangle\right.$ et on a vu qu'il existe $\tau_{1}, \tau_{2}$ des séries discrètes irréductibles de $U\left(n_{1}, E / F\right)$ et $U\left(n_{2}, E / F\right)$ respectivement telles que

$$
\pi=\pi_{\tau_{1}} \times\left(\omega \otimes \pi_{\tau_{2}}\right) .
$$

On écrit $\pi=\times_{\left(\rho^{\prime}, a^{\prime}\right) \in \mathscr{E}} \operatorname{St}\left(\rho^{\prime}, a^{\prime}\right)$ et pour $i=1,2$,

$$
\pi_{\tau_{i}}=\underset{\left(\rho^{\prime}, a^{\prime}\right) \in \mathscr{E}_{i}}{\times} \operatorname{St}\left(\rho^{\prime}, a^{\prime}\right) .
$$

On sait que $\pi=\pi_{\tau_{1} \otimes \tau_{2}}$ ce qui est équivalent à dire que

$$
\mathscr{E}=\mathscr{E}_{1} \bigcup_{\left(\rho^{\prime}, a^{\prime}\right) \in \mathscr{E}_{2}}\left(\omega \rho^{\prime}, a^{\prime}\right) .
$$

Or soit $\left(\rho^{\prime}, a^{\prime}\right) \in \mathscr{E}$; alors $\left(\rho^{\prime}, a^{\prime}\right)$ ne peut être dans $\mathscr{E}_{1}$ que si $a^{\prime}$ est pair quand $L\left(\rho^{\prime}, r_{A}^{\prime}, s\right)$ n'a pas de pôle en $s=0$ et impair sinon. Et $\left(\omega \rho^{\prime}, a^{\prime}\right)$ ne peut être dans $\mathscr{E}_{2}$ que si $a^{\prime}$ est pair quand $L\left(\omega \rho^{\prime}, r_{A}^{\prime}, s\right)$ a un pôle en $s=0$ et impair sinon. Les propriétés de la fonction $L\left(\rho^{\prime}, r_{A}^{\prime}, s\right)$ et la paritié de $a^{\prime}$ détermine donc si $\left(\rho^{\prime}, a^{\prime}\right) \in$ $\mathscr{E}_{1}$ ou si $\left(\omega \rho^{\prime}, a^{\prime}\right) \in \mathscr{E}_{2}$ les 2 possibilités étant exclusives l'une de l'autre. D'où l'unicité de $\langle H\rangle$ et le théorème.

\section{Cardinal des paquets stables}

On suppose ici que $U(n, E / F)$ est quasidéployé.

On fixe un morphisme $\psi$ de $W_{E} \times \operatorname{SL}(2, \mathbb{C})$ dans $\operatorname{GL}(n, \mathbb{C}), \theta$-discret et stable ; on a défini le paquet de séries discrètes $\Pi(\psi)$. On note $I_{\text {cusp }}^{U}[\psi]$ le sous-espace vectoriel de $I_{\text {cusp }}(U(n, E / F))$ engendré par l'image des éléments de $\Pi(\psi)$. Soit 
$H$ un groupe endoscopique de $U(n, E / F) ; H$ est de la forme $U\left(n_{1}, E / F\right) \times$ $U\left(n_{2}, E / F\right)$ avec $n_{1}+n_{2}=n$ et l'ordre ici n'importe pas. En particulier si $n_{1}=$ $n_{2}$, la donnée endoscopique qui vient avec $H$ admet un automorphisme extérieur provenant de $U(n)$. Pour $n_{1}, n_{2}$ tel que $n_{1}+n_{2}=n$, on définit d'abord $I_{\text {cusp }}^{n_{1}, n_{2}}[\psi]$ en généralisant la définition ci-dessus et en sommant sur tous les morphismes, $\psi^{\prime}$ de $W_{E} \times \operatorname{SL}(2, \mathbb{C})$ dans $\operatorname{GL}\left(n_{1}, \mathbb{C}\right) \times \operatorname{GL}\left(n_{2}, \mathbb{C}\right)$ dont l'image par l'inclusion de $\operatorname{GL}\left(n_{1}, \mathbb{C}\right) \times \operatorname{GL}\left(n_{2}, \mathbb{C}\right)$ dans $\operatorname{GL}(n, \mathbb{C})$ est conjugué de $\psi$. Et on note $I_{\text {cusp }}^{n_{1}, n_{2}, \text { st }}[\psi]$ le sous-espace de $I_{\text {cusp }}^{n_{1}, n_{2}}[\psi]$ formé des éléments stables.

Ici on ne peut plus ignorer le fait que certaines représentations elliptiques ne sont pas des séries discrètes. On note $I_{\text {cusp }}^{n \text {,ell }}$ le sous-espace vectoriel de $I_{\text {cusp }}(U(n, E / F)$ engendré par l'image des représentations elliptiques qui ne sont pas des séries discrètes. On a déjà vu en 5.7 que

$$
I_{\text {cusp }}(U(n, E / F))=\bigoplus_{\psi} I_{\text {cusp }}[\psi] \oplus I_{\text {cusp }}^{n, \text { ell }},
$$

où $\psi$ parcourt l'ensemble des morphismes $\theta$-discrets pris à conjugaison près somme de représentations stables. Donc

$$
I_{\text {cusp }}^{\text {st }}(U(n, E / F))=\bigoplus_{\psi} \mathbb{C} f_{\psi},
$$

où $f_{\psi}$ est la combinaison linéaire stable de séries discrètes dans $\Pi(\psi)$ (voir encore 5.7). On a en plus

$$
I_{\text {cusp }}^{n, \text { ell }} \cap I_{\text {cusp }}^{\text {st }}(U(n, E / F))=0,
$$

puisque $I_{\text {cusp }}^{\text {st }}\left(U(n, E / F)=I_{\text {cusp }}^{\text {st }}\left(\tilde{G}_{n}\right)\right.$ a été décrit par comme étant égal à (7-1).

Pour $n=n_{1}+n_{2}$ comme ci-dessus avec $n_{1} n_{2} \neq 0$, on définit différemment $I_{\text {cusp }}^{n_{1}, n_{2}, \text { st ell }}$. On pose :

$$
I_{\text {cusp }}^{n_{1}, n_{2}, \text { st,ell }}:=\bigoplus_{\psi_{1} \times \psi_{2}} I_{\text {cusp }}^{\text {st }}\left(U\left(n_{1}, E / F\right) \times U\left(n_{2}, E / F\right)\right)\left[\psi_{1} \times \psi_{2}\right],
$$

où la somme porte sur les morphismes $\psi_{i}$ (pour $\left.i=1,2\right)$ de $W_{E} \times \operatorname{SL}(2, \mathbb{C})$ dans $\operatorname{GL}\left(n_{i}, \mathbb{C}\right), \theta$-discrets mais tels que les représentations définies par $\psi_{1}$ et $\psi_{2}$ ne sont pas disjointes. En d'autres termes $\psi_{1} \times \psi_{2}$ vu comme représentation de dimension $n$ n'est pas $\theta$-discrète.

On utilise la décomposition de [Arthur 1996, 3.5] ; c'est ici que l'on utilise le fait que $U(n, E / F)$ est quasidéployé :

$$
I_{\text {cusp }}(U(n, E / F))=\bigoplus_{n_{1}, n_{2}} I_{\text {cusp }}^{\text {st,Out }}\left(U\left(n_{1}, E / F\right) \times U\left(n_{2}, E / F\right)\right),
$$

où la somme porte sur les couples non ordonnés $n_{1}, n_{2}$ tel que $n=n_{1}+n_{2}$ et où Out indique que si $n_{1}=n_{2}$ on considère les éléments invariants par l'action de l'automorphisme extérieur échangeant les 2 copies. Si $n_{1} \neq n_{2}$, Out est sans objet. Rappelons qu'ici on a supposé $U(n, E / F)$ quasi-déployé. 
Soit $\psi$ comme ci-dessus et $n_{1}=n_{2}$; l'espace $I_{\text {cusp }}^{n_{1}, n_{2}, \mathrm{st}}[\psi]$ est stable sous-l'action de Out. Son supplémentaire naturel (l'espace des éléments instables, c'est-à-dire les intégrales orbitales dont la somme sur toute classe stable est nulle) est lui aussi stable sous Out et on obtient facilement :

$$
\begin{aligned}
& I_{\text {cusp }}^{\text {st, Out }}\left(U\left(n_{1}, E / F\right) \times U\left(n_{2}, E / F\right)\right)= \\
& \sum_{\psi} I_{\text {cusp }}^{\text {st, Out }}\left(U\left(n_{1}, E / F\right) \times U\left(n_{2}, E / F\right)\right)[\psi] \oplus I_{\text {cusp }}^{\text {st,ell,Out }}\left(U\left(n_{1}, E / F\right) \times U\left(n_{2}, E / F\right)\right) .
\end{aligned}
$$

Lemme. Soit $\psi$ un morphisme $\theta$-discret comme ci-dessus. Soit $n=n_{1}+n_{2}$.

L'image de $I_{\text {cusp }}^{\text {st, Out }}\left(U\left(n_{1}, E / F\right) \times U\left(n_{2}, E / F\right)\right)[\psi]$ dans $I_{\text {cusp }}(U(n, E / F))$ suivant (7-2) est incluse dans $I_{\text {cusp }}(U(n, E / F))[\psi]$ et vice et versa.

Etant donné les décompositions en somme directe utilisant les morphismes $\psi$ déjà écrites, il suffit de montrer l'assertion suivante :

on fixe $\tau \in \Pi(\psi)$ et on montre que la décomposition de $\tau$ vu comme élément de $I_{\text {cusp }}(U(n, E / F))$ a une projection sur chaque $I_{\text {cusp }}^{\text {st }}\left(U\left(n_{1}, E / F\right) \times U\left(n_{2}, E / F\right)\right)$ incluse dans

$$
I_{\text {cusp }}^{\text {st }}\left(U\left(n_{1}, E / F\right) \times U\left(n_{2}, E / F\right)\right)[\psi] .
$$

Démontrons cela. On écrit $f_{\tau}$ l'élément de $I_{\text {cusp }}(U(n, E / F))$ correspondant à $\tau$. Et on décompose :

$$
f_{\tau}=\bigoplus_{H} f_{\tau}^{H},
$$

où pour tout $H$ groupe endoscopique elliptique de $U(n, E / F), f_{\tau}^{H}$ est un élément de $I_{\text {cusp }}^{\text {st }}\left(U\left(n_{1}, E / F\right) \times U\left(n_{2}, E / F\right)\right)$. On peut donc décomposer cet élément suivant la base obtenue à l'aide du produit de morphisme $\psi_{1} \times \psi_{2}$, où $\psi_{1}$ et $\psi_{2}$ sont $\theta$-discrets et on doit démontrer que seuls interviennent les morphismes $\psi_{1} \times \psi_{2}$ qui sont conjugués de $\psi$. C'est donc encore un problème de support cuspidal; il faut démontrer que le support cuspidal étendu de $\tau$ est le support cuspidal de $\pi\left(\psi_{1}\right) \times \pi\left(\psi_{2}\right)$.

On reprend les méthodes déjà utilisées; on écrit

$$
\pi(\psi)=\underset{(\rho, a) \in \mathscr{E}}{\times} \operatorname{St}(\rho, a) .
$$

On note $\psi^{+}$le morphisme tel que

$$
\pi\left(\psi^{+}\right)=\underset{(\rho, a) \in \mathscr{E}}{\times} \operatorname{St}(\rho, a+2) .
$$

Et on a montré dans la preuve de la proposition de 5.4, l'existence d'un élément $\tau^{+}$dans $\Pi\left(\psi^{+}\right)$sous-module irréductible de l'induite

$$
\underset{(\rho, a) \in \mathscr{E}}{\times} \rho \mid \|_{E}^{(a+1) / 2} \times \tau
$$


où ici l'ordre sur $\mathscr{E}$ est important; l'élément $(\rho, a)$ précède (c'est -à-dire est à gauche ci-dessus) l'élément $\left(\rho, a^{\prime}\right)$ si $a<a^{\prime}$.

On écrit la décomposition de $f_{\tau^{+}}$comme ci-dessus et on calcule les modules de Jacquet par rapport aux éléments $\rho \|_{E}^{(a+1) / 2}$ pris dans le même ordre : on commence par celui de gauche et on "remonte". Pour $H$ fixé, $\psi_{1} \times \psi_{2}$ et $\tau_{1} \otimes \tau_{2}$ une série discrète de ce paquet, intervenant dans la décomposition de $f_{\tau}^{H}$, on trouve qu'il existe (au moins) un groupe endoscopique $H_{1}^{\prime} \times H_{2}^{\prime}$, (au moins) un morphisme $\psi_{1}^{\prime} \times \psi_{2}^{\prime}$ et (au moins) une série discrète, $\tau_{1,2}^{\prime}$ dans le paquet défini, dont le module de Jacquet contient comme sous-quotient

$$
\left(\underset{\left(\rho^{\prime}, a^{\prime}\right) \in \mathscr{E}_{1}}{\otimes} \rho^{\prime}||_{E}^{\left(a^{\prime}+1\right) / 2} \otimes \tau_{1}\right) \otimes\left(\underset{\left(\rho^{\prime \prime}, a^{\prime \prime}\right) \in \mathscr{E}_{2}}{\otimes} \rho^{\prime \prime}||_{E}^{\left(a^{\prime \prime}+1\right) / 2} \otimes \tau_{2}\right),
$$

où la décomposition de $\mathscr{E}$ en $\mathscr{E}_{1} \cup \mathscr{E}_{2}$ dépend des choix et ne nous importe pas. En prenant les supports cuspidaux étendus, on trouve :

$\operatorname{Supp} \operatorname{cusp}\left(\pi\left(\psi_{1}^{\prime}\right) \times \pi\left(\psi_{2}^{\prime}\right)\right)$

$$
=\operatorname{Supp} \operatorname{cusp}\left(\pi\left(\psi_{1}\right) \times \pi\left(\psi_{2}\right)\right) \cup\left\{\rho||^{(a+1) / 2}, \rho||^{-(a+1) / 2}\right\} .
$$

Ici on a été un peu trop vite en faisant toutes les étapes en un seul coup mais elle sont progressives : ce que l'on veut est que les éléments $(\rho,(a+1) / 2),(\rho,-(a+1) / 2$ sont des extrémités des segments de $\psi_{1}^{\prime} \times \psi_{2}^{\prime}$. On a déjà vu dans la preuve de la proposition de 5.4 qu'il en était bien ainsi. On a aussi vu qu'un simple calcul des dimensions assurent que tout segment dans le support cuspidal de $\pi\left(\psi_{1}^{\prime}\right) \times \pi\left(\psi_{2}^{\prime}\right)$ est obtenu ainsi. On en déduit donc que le support cuspidal de $\pi\left(\psi_{1}^{\prime}\right) \times \pi\left(\psi_{2}^{\prime}\right)$ est précisément celui de la représentation :

$$
\underset{(\rho, a) \in \mathscr{E}}{\times} \operatorname{St}(\rho, a+2) .
$$

Et celui de $\pi\left(\psi_{1}\right) \times \pi\left(\psi_{2}\right)$ s'obtient en enlevant toutes les extrémités et c'est donc celui de $\pi(\psi)$ comme annoncé.

\subsection{Cardinal.}

Théorème. Soit $\psi: W_{E} \times \mathrm{SL}(2, \mathbb{C}) \rightarrow \mathrm{GL}(n, \mathbb{C})$ un morphisme $\theta$-discret et stable. On note $\ell(\psi)$ la longueur de la représentation ainsi définie. Alors:

$$
|\Pi(\psi)|=2^{\ell(\psi)-1} .
$$

On sait que le nombre d'éléments dans $\Pi(\psi)$ est exactement la dimension de l'espace vectoriel $I_{\text {cusp }}\left(U(n, E / F)[\psi]\right.$. Et on sait aussi que $I_{\text {cusp }}^{\text {st }}[\psi]$ est de dimension 1. Soit $n_{1}+n_{2}=n$ une décomposition ordonnée de $n$ et soit $\psi_{i}$ pour $i=1,2$ des morphismes de $W_{E} \times \operatorname{SL}(2, \mathbb{C})$ dans $\operatorname{GL}\left(n_{i}, \mathbb{C}\right)$. L'automorphisme de 


$$
\begin{aligned}
& I_{\text {cusp }}\left(U\left(n_{1}, E / F\right) \times U\left(n_{2}, E / F\right)\right)\left[\psi_{1} \times \psi_{2}\right] \operatorname{sur} \\
& I_{\text {cusp }}\left(U\left(n_{2}, E / F\right) \times U\left(n_{1}, E / F\right)\right)\left[\psi_{2} \times \psi_{1}\right]
\end{aligned}
$$

envoie la droite "stable" sur son homologue. On suppose que $\psi_{1} \times \psi_{2}$ est conjugué de $\psi$; alors $\psi_{1}$ et $\psi_{2}$ n'ont aucune sous-représentation en commun. Cela prouve que si $n$ est pair, le groupe Out n'a pas de droite fixe dans $I_{\text {cusp }}^{\text {st }}(U(n / 2, E / F) \times$ $U(n / 2, E / F))[\psi]$ d'où

$\operatorname{dim} I_{\text {cusp }}^{\text {st,Out }}(U(n / 2, E / F) \times U(n / 2, E / F))[\psi]$

$$
=1 / 2 \operatorname{dim} I_{\text {cusp }}^{\mathrm{st}}(U(n / 2, E / F) \times U(n / 2, E / F))[\psi] .
$$

On a donc

(7-3) $\operatorname{dim} I_{\text {cusp }}(U(n, E / F))[\psi]$

$$
=1 / 2 \sum_{\substack{\left(n_{1}, n_{2}\right) \\ n_{1}+n_{2}=n}} \operatorname{dim} I_{\text {cusp }}^{\mathrm{st}}\left(U\left(n_{1}, E / F\right) \times U\left(n_{2}, E / F\right)\right)[\psi],
$$

où la somme porte sur les décompositions ordonnées de $n$; entre autre la décomposition $(0, n)$ n'est pas la même que $(n, 0)$. Soit $n_{1}, n_{2}$ une décomposition de $n$. On a encore

$$
\begin{aligned}
I_{\text {cusp }}^{\text {st }}\left(U\left(n_{1}, E / F\right) \times U\right. & \left.\left(n_{2}, E / F\right)\right)[\psi] \\
& =\bigoplus_{\psi_{1}, \psi_{2}} I_{\text {cusp }}^{\text {st }}\left(U\left(n_{1}, E / F\right)\right)\left[\psi_{1}\right] \otimes I_{\text {cusp }}^{\text {st }}\left(U\left(n_{2}, E / F\right)\right)\left[\psi_{2}\right],
\end{aligned}
$$

où $\psi_{1}, \psi_{2}$ sont comme ci-dessus et $\psi_{1} \times \psi_{2}$ est conjugué de $\psi$. Il faut donc calculer le nombre de telles décompositions. Pour faire ces calculs, on inverse les sommes : on décompose $\psi$ en le produit de 2 représentations de $W_{E} \times \operatorname{SL}(2, \mathbb{C})$, produit ordonné, et cette décomposition détermine uniquement $n_{1}$ et $n_{2}$. Ces décompositions sont en bijection avec l'ensemble des applications de l'ensemble des sousreprésentations irréductibles incluses dans $\psi$ dans $\{ \pm 1\}$. Il y a donc $2^{\ell(\psi)}$ telles décompositions. Il ne faut pas oublier le 1/2 de (7-3) ci-dessus et on trouve le résultat.

\section{Classification des représentations cuspidales}

8.1. Définitions. On fixe un homomorphisme $\psi$ de $W_{E} \times \operatorname{SL}(2, \mathbb{C})$ dans $\operatorname{GL}(n, \mathbb{C})$, $\theta$ discret et on suppose que ce morphisme paramétrise un paquet non vide, $\Pi(\psi)$ de séries discrètes. Soit $s$ un élément, $\theta$-invariant, du centralisateur de $\psi$ dans $\operatorname{GL}(n, \mathbb{C})$. On note $z$ la matrice diagonale de $\operatorname{GL}(n, \mathbb{C})$ de valeurs propres -1 . Le centralisateur de $s$ dans $\operatorname{GL}(n, \mathbb{C})$ est un produit de 2 groupes linéaires $\operatorname{GL}\left(n_{1}, \mathbb{C}\right) \times$ $\mathrm{GL}\left(n_{2}, \mathbb{C}\right)$, où $n_{1}$ est la dimension de l'espace propre correspondant à la valeur propre -1 de $s$. En remplaçant $s$ par $z s$ on échange $n_{1}$ et $n_{2}$. Puisque $s$ est dans 
le centralisateur de $\psi$, le morphisme $\psi$ se factorise par $\operatorname{GL}\left(n_{1}, \mathbb{C}\right) \times \operatorname{GL}\left(n_{2}, \mathbb{C}\right)$ et on note $\psi_{s}$ cette factorisation. On obtient ainsi une représentation $\pi\left(\psi_{s}\right)$ de $\operatorname{GL}\left(n_{1}, E\right) \times \operatorname{GL}\left(n_{2}, E\right)$. On la prolonge en une représentation de $\tilde{G}_{n_{1}} \times \tilde{G}_{n_{2}}$ ce qui donne naturellement un élément de $I_{\text {cusp }}^{\text {st }}\left(\tilde{G}_{n_{1}} \times \tilde{G}_{n_{2}}\right)$ et donc de $I_{\text {cusp }}^{\text {st }}\left(U\left(n_{1}, E / F\right) \otimes\right.$ $I_{\text {cusp }}^{\text {st }}\left(U\left(n_{2}, E / F\right)\right.$. On note $\Psi_{s}$. la projection de cet élément dans $I_{\text {cusp }}^{\text {st, Out }}(H)$, où $H$ est la donnée endoscopique de $U(n, E / F)$ associée au groupe $U\left(n_{1}, E / F\right) \times$ $U\left(n_{2}, E / F\right)$. Comme nous n'avons pas fixé le choix de l'extension, en travaillant avec $s z$ plutôt que $s$ on aurait trouvé le même résultat au signe près. On fixe donc un choix et on note $f_{\psi, s}$ l'image de cet élément dans $I_{\text {cusp }}(U(n, E / F))$. On a vu que cet élément s'interprète comme une combinaison linéaire des caractères des représentations dans $\Pi(\psi)$. Ainsi par inversion, on définit pour tout élément $\pi \in \Pi(\psi)$ des nombres complexes $d\left(s^{*}, \pi\right)$ uniquement déterminés par l'égalité de caractères :

$$
\operatorname{tr} \pi=\sum_{(s, s z)} d\left(s^{\cdot}, \pi\right) f_{\psi, s} .
$$

On décompose $\psi$ en représentations irréductibles et soit $(\rho, a)$ un couple formé d'une représentation irréductible de $W_{E}$ et d'un entier $a$ tel que, en notant $\sigma_{[a]}$ la représentation irréductible de $\operatorname{SL}(2, \mathbb{C})$ de dimension $a$, la représentation $\rho \otimes \sigma_{[a]}$ de $W_{E} \times \operatorname{SL}(2, \mathbb{C})$ soit une sous-représentation de $\psi$. On suppose qu'il existe $0 \leq b<a$ tel que, si $b \neq 0, \rho \otimes \sigma_{[b]}$ soit aussi une sous-représentation de $\psi$ et si $b=0$ que $a$ est pair. On note alors $a_{-}$le plus grand élément $b$ vérifiant les propriétés ci-dessus. On pose $z_{\rho, a}$ l'élément du centralisateur de $\psi$ dans $\operatorname{GL}(n, \mathbb{C})$ dont les valeurs propres -1 ont exactement pour espace propre la somme de $\rho \otimes \sigma_{[a]} \oplus \rho \otimes \sigma_{\left[a_{-}\right]}$. On note $A(\psi)$ le sous-groupe du centralisateur de $\psi$ engendré par ces éléments $z_{\rho, a}$ quand $(\rho, a)$ parcourt tous les couples possibles.

Lemme. Soient $(\rho, a), z_{\rho, a}$ ayant les propriétés précédentes. Pour tout s comme ci-dessus, il existe un signe $\zeta_{s, \rho, a}$ tel que

$$
\operatorname{Jac}_{\rho \|\left.|(a-1) / 2, \ldots, \rho|\right|^{\left(a_{-}+1\right) / 2}} f_{\psi, s}=\zeta_{s, \rho, a} \operatorname{Jac}_{\rho\|\mid(a-1) / 2, \ldots, \rho\|^{\left(a_{-}+1\right) / 2}} f_{\psi, s z_{\rho, a}} .
$$

Il faut distinguer deux cas. Dans le premier cas l'espace propre pour la valeur propre -1 de $s$ contient l'espace de la représentation $\rho \otimes \sigma_{[a]}$ et ne contient pas l'espace de la représentation $\rho \otimes \sigma_{\left[a_{-}\right]}$. Le deuxième cas est le cas où la valeur propre -1 de $s$ a un espace propre qui contient la somme de ces 2 représentations. Quitte à changer $s$ en $s z$, on se trouve dans l'un ou l'autre cas.

Dans les deux cas, la démonstration utilise le fait que prendre les modules de Jacquet est compatible avec le transfert endoscopique. Pour simplifier l'écriture, on enlève les $E$ des valeurs absolues. On a défini $\operatorname{Jac}_{\sigma}^{\theta}$ à la fin de 1.6 pour $\sigma$ une représentation cuspidale ; on généralise cette notation à un ensemble fini de représentations cuspidales, $\sigma_{1}, \ldots, \sigma_{k}$, en posant $\mathrm{Jac}_{\sigma_{1}, \ldots, \sigma_{k}}^{\theta}:=\mathrm{Jac}_{\sigma_{k}}^{\theta} \circ \cdots \circ \mathrm{Jac}_{\sigma_{1}}^{\theta}$. 
Considérons d'abord le premier cas ; on calcule $\operatorname{Jac}_{\rho\left\|^{(a-1) / 2}, \ldots, \rho\right\|^{\left(a_{-}+1\right) / 2}}^{\theta} \pi\left(\psi_{s}\right)$; on écrit $\psi_{s}=\psi_{1} \times \psi_{2}$ où $\psi_{i}$ pour $i=1,2$ est à valeurs dans $\operatorname{GL}\left(n_{i}, \mathbb{C}\right)$, décomposition suivant les espaces propres de $s$. On a alors facilement :

$\operatorname{Jac}_{\rho||(a-1) / 2, \ldots, \rho||^{\left(a_{-}+1\right) / 2}}^{\theta}\left(\pi\left(\psi_{1}\right) \otimes \pi\left(\psi_{2}\right)\right)$

$$
=\left(\operatorname{Jac}_{\rho||^{(a-1) / 2}, \ldots, \rho||^{\left(a_{-}+1\right) / 2}}^{\theta} \pi\left(\psi_{1}\right)\right) \otimes \pi\left(\psi_{2}\right) .
$$

On note $\psi_{1}^{\prime}$ le morphisme qui se déduit de $\psi_{1}$ en remplaçant la sous-représentation $\rho \otimes \sigma_{[a]}$ par $\rho \otimes \sigma_{\left[a_{-}\right]}$et le résultat est $\pi\left(\psi_{1}^{\prime}\right) \otimes \pi\left(\psi_{2}\right)$. L'action de $\theta$ sur le résultat est déterminée par le choix fait au départ.

On fait le même calcul en partant de $s z_{\rho, a}$. Alors le morphisme $\psi_{s z_{\rho, a}}$ vaut $\psi_{1}^{\prime} \otimes \psi_{2}^{\prime}$ où $\psi_{1}^{\prime}$ est exactement comme ci-dessus et $\psi_{2}^{\prime}$ se déduit de $\psi_{2}$ en remplaçant la représentation $\rho \otimes \sigma_{\left[a_{-}\right]}$en $\rho \otimes \sigma_{[a]}$. Et on obtient :

$$
\begin{aligned}
& \operatorname{Jac}_{\rho||^{(a-1) / 2}, \ldots, \rho||^{\left(a_{-}+1\right) / 2}}^{\theta}\left(\pi\left(\psi_{1}^{\prime}\right) \otimes \pi\left(\psi_{2}^{\prime}\right)\right)=\pi\left(\psi_{1}^{\prime}\right) \otimes\left(\operatorname{Jac}_{\rho||^{(a-1) / 2}, \ldots,\left.\rho\right|^{\left(a_{-}+1\right) / 2}}^{\theta} \pi\left(\psi_{2}^{\prime}\right)\right) \\
& =\pi\left(\psi_{1}^{\prime}\right) \otimes \pi\left(\psi_{2}\right) .
\end{aligned}
$$

Il n'y a pas de difficulté à faire agir le groupe des automorphismes extérieurs venant de $U(n, E / F)$ sur la donnée endoscopique pour garder l'égalité au signe près des transferts endoscopiques

$$
\operatorname{Jac}_{\rho||(a-1) / 2, \ldots, \rho \|^{\left(a_{-}+1\right) / 2}} f_{\psi, s} \quad \text { et } \quad \operatorname{Jac}_{\rho||^{(a-1) / 2}, \ldots, \rho \|^{\left(a_{-}+1\right) / 2}} f_{\psi, s z_{\rho, a}},
$$

le signe dépend de l'action de $\theta$; ici c'est une égalité dans

$$
I_{\text {cusp }}\left(U\left(n-\left(a-a_{-}\right) d_{\rho}, E / F\right)\right),
$$

on se trouve dans la partie elliptique non série discrète.

Considérons maintenant le deuxième cas : on note encore $\psi_{1} \times \psi_{2}$ la décomposition en produit de $\psi_{s}$. Ici on note $\psi_{1}^{\prime}$ le morphisme qui se déduit de $\psi_{1}$ en enlevant les 2 représentations $\rho \otimes \sigma_{[a]}$ et $\rho \otimes \sigma_{\left[a_{-}\right]}$. Et on a :

$$
\begin{aligned}
\operatorname{Jac}_{\rho||^{(a-1) / 2}, \ldots, \rho||^{\left(a_{-}+1\right) / 2}}^{\theta}\left(\pi\left(\psi_{1}\right) \otimes \pi\left(\psi_{2}\right)\right) & \\
& =\left(\operatorname{Jac}_{\rho||^{(a-1) / 2}, \ldots, \rho||^{\left(a_{-}+1\right) / 2}}^{\theta} \pi\left(\psi_{1}\right)\right) \otimes \pi\left(\psi_{2}\right) \\
& =\left(\operatorname{St}\left(\rho, a_{-}\right) \times \operatorname{St}\left(\rho, a_{-}\right) \times \pi\left(\psi_{1}^{\prime}\right)\right) \otimes \pi\left(\psi_{2}\right) .
\end{aligned}
$$

Il y a en plus une action de $\theta$ que l'on n'écrit pas. Ici on quitte les espaces $I_{\text {cusp }}$ à cause du fait que $\operatorname{St}(\rho, a)$ intervient deux fois. Mais on sait calculer le transfert vers une distribution stable de $U\left(n_{1}-\left(a-a_{-}\right) d_{\rho}, E / F\right) \times U\left(n_{2}, E / F\right)$, cela vaut exactement

$$
\operatorname{St}\left(\rho, a_{-}\right) \times \operatorname{Transfert}^{\mathrm{st}}\left(\pi\left(\psi_{1}^{\prime}\right) \otimes \pi\left(\psi_{2}\right)\right)
$$

où le Transfert ${ }^{\text {st }}$ est le transfert stable réciproque entre $\tilde{G}_{n_{1}^{\prime}} \times \tilde{G}_{n_{2}}$ et le produit de groupe unitaire correspondant $\left(n_{1}^{\prime}\right.$ est la dimension de la représentation $\psi_{1}^{\prime}$ et $n_{2}$ 
celle de $\left.\psi_{2}\right)$. Il faut maintenant faire le transfert endoscopique vers $U(n, E / F)$; ce transfert est compatible à l'induction $\operatorname{par} \operatorname{St}\left(\rho, a_{-}\right)$et son image est donc

$$
\operatorname{St}\left(\rho, a_{-}\right) \times \pi^{H}\left(\psi_{1}^{\prime} \times \psi_{2}\right)
$$

où $\pi^{H}\left(\psi_{1}^{\prime} \times \psi_{2}\right)$ est le transfert endoscopique de $\operatorname{Transfert}^{\text {st }}\left(\pi\left(\psi_{1}^{\prime}\right) \otimes \pi\left(\psi_{2}\right)\right)$; pour calculer cette représentation virtuelle, on peut de nouveau se placer dans le $I_{\text {cusp }}$ convenable et on obtient un élément de la forme $f_{\psi_{1}^{\prime} \times \psi_{2}, s^{\prime}}$, où $s^{\prime}$ est la restriction de $s$ sur l'espace de $\psi_{1}^{\prime} \times \psi_{2}$.

On fait le même calcul en partant de $s z_{\rho, a}$; la décomposition de $\psi_{s}$ est $\psi_{1}^{\prime} \times \psi_{2}^{\prime}$, où $\psi_{1}^{\prime}$ est comme ci-dessus et $\psi_{2}^{\prime}$ est la somme de $\psi_{2}$ avec les 2 représentations $\rho \otimes \sigma_{[a]}$ et $\rho \otimes \sigma_{\left[a_{-}\right]}$. Les calculs se font ensuite exactement comme ci-dessus, c'est $\psi_{1}^{\prime}$ qui joue un rôle muet et $\pi\left(\psi_{2}\right)$ dont on prend le module de Jacquet. On obtient encore

$$
\begin{aligned}
\operatorname{Jac}_{\rho||^{(a-1) / 2}, \ldots, \rho||^{\left(a_{-}+1\right) / 2}}^{\theta}\left(\pi\left(\psi_{1}^{\prime}\right) \otimes \pi\left(\psi_{2}^{\prime}\right)\right) & \\
& =\pi\left(\psi_{1}^{\prime}\right) \otimes\left(\operatorname{Jac}_{\left.\rho\right|^{(a-1) / 2}, \ldots, \rho||^{\left(a_{-}+1\right) / 2}}^{\theta} \pi\left(\psi_{2}^{\prime}\right)\right) \\
& =\pi\left(\psi_{1}^{\prime}\right) \otimes\left(\operatorname{St}\left(\rho, a_{-}\right) \times \operatorname{St}\left(\rho, a_{-}\right) \times \pi\left(\psi_{2}\right)\right) .
\end{aligned}
$$

La fin est exactement comme ci-dessus, on commence par sortir l'induction puis on peut retravailler dans $I_{\text {cusp. }}$. On obtient encore comme image $\operatorname{St}\left(\rho, a_{-}\right) \times f_{\psi_{1}^{\prime} \times \psi_{2}, s^{\prime}}$.

Cela prouve le lemme.

8.2. Traduction des propriétés des modules de Jacquet. Fixons $\psi$ un morphisme de $W_{E} \times \operatorname{SL}(2, \mathbb{C})$ dans $\operatorname{GL}(n, \mathbb{C}), \theta$-discret et stable.

On a déjà défini les fonctions $d\left(\tau, s^{*}\right)$ et les signes $\zeta_{s, \rho, a}$. On a vu que pour $s=1, d\left(\tau, 1^{\cdot}\right) \neq 0$ pour tout $\tau \in \Pi(\psi)$; c'est le fait que la projection de $\operatorname{tr} \tau$ sur $I_{\text {cusp }}^{\text {st }}(U(n, E / F)$ est non nulle. La première des conjectures est que pour un bon choix de l'extension de $\pi(\psi)$ à $\tilde{G}_{n}$, pour tout $\tau, d\left(\tau, 1^{\cdot}\right)$ vaut $|\Pi(\psi)|^{-1}$, c'està-dire $2^{-\ell(\psi)+1}$. En choisissant la même normalisation pour tous les groupes $\tilde{G}_{m}$ pour $m \leq n$, on normalise ainsi tous les éléments qui ont servi à définir la matrice $d\left(\tau, s^{*}\right)$. Les conjectures d'Arthur sont exprimées en termes de la matrice inverse de la matrice $\left(d\left(\tau, s^{*}\right)\right)$. En traduisant cela donne :

$d\left(\tau, s^{*}\right) 2^{\ell(\psi)-1}$ est un signe et l'application $s \mapsto d\left(\tau, s^{*}\right) 2^{\ell(\psi)-1}$ est un caractère de $\left(\operatorname{Cent}_{\mathrm{GL}(n, \mathbb{C})} \psi\right)^{\theta}$ trivial sur le sous-groupe du centre de $\operatorname{GL}(n, \mathbb{C})$ formé des éléments $\theta$-invariants.

Une variante plus faible serait de montrer que pour tout $s, s^{\prime}$ dans le centralisateur, $d(s, \tau) d\left(s^{\prime}, \tau\right) \neq 0$ et le quotient $d(s, \tau) / d\left(s^{\prime}, \tau\right)$ ne dépend que de $s s^{\prime}($ tout élément du centralisateur est de carré 1). Nous allons montrer une forme plus faible encore mais qui suffit pour caractériser les représentations cuspidales. Soit 
$(\rho, a)$ comme dans 8.1 avec $a_{-}$défini. On prend la notation :

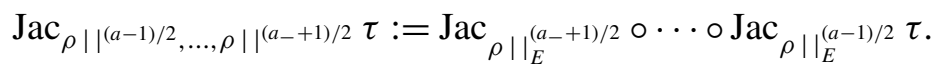

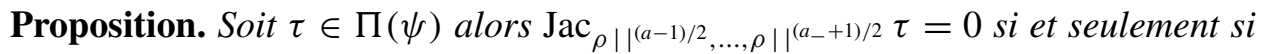
pour tout s comme ci-dessus

$$
d\left(s^{\cdot}, \tau\right)=-\zeta_{s, \rho, a} d\left(s^{*} z_{\rho, a}, \tau\right)
$$

On simplifie la notation en remplaçant $\mathrm{Jac}_{\left.\rho||\right|^{(a-1) / 2}, \ldots, \rho \|^{\left(a_{-}+1\right) / 2}} \mathrm{par}$

$$
\operatorname{Jac}_{(a-1) / 2, \ldots,\left(a_{-}+1\right) / 2} .
$$

On écrit avec les définitions, pour tout $\tau \in \Pi(\psi)$ et pour $\rho, a$ comme ci-dessus :

$$
\begin{aligned}
\operatorname{Jac}_{(a-1) / 2, \ldots,\left(a_{-}+1\right) / 2} & \tau \\
& =\sum_{s \cdot} d\left(s^{\cdot}, \tau\right) \operatorname{Jac}_{(a-1) / 2, \ldots,\left(a_{-}+1\right) / 2} f_{\psi, s} \\
& =\sum_{\left(s \cdot, \cdot z_{\rho, a}\right)}\left(d\left(s^{\prime}, \tau\right)+\zeta_{s, \rho, a} d\left(s^{\prime} z_{\rho, a}, \tau\right)\right) \operatorname{Jac}_{(a-1) / 2, \ldots,\left(a_{-}+1\right) / 2} f_{\psi, s} .
\end{aligned}
$$

Il est clair que si la condition de l'énoncé est vérifiée, cette somme est nulle terme à terme. C'est la réciproque qu'il faut prouver à savoir partir de $\tau$ avec $\operatorname{Jac}_{(a-1) / 2, \ldots,\left(a_{-}+1\right) / 2} \tau=0$ et en déduire que chaque terme de la somme est nulle. On reprend les calculs plus précis des termes

$$
\operatorname{Jac}_{(a-1) / 2, \ldots,\left(a_{-}+1\right) / 2} f_{\psi, s}
$$

faits dans la preuve du lemme de 8.1. On réutilise la filtration introduite dans [Arthur 1996]; le terme de plus "haut" degré est ce qui provient de $I_{\text {cusp }}(U(n, E / F))$ et les autres termes de la filtration ont un gradué qui voit les $I_{\text {cusp }}(M)$ pour $M$ les Levi de $U(n, E / F)$. On projette le membre de droite de l'égalité ci-dessus sur le plus haut terme de cette filtration; toutes les contributions des $s$ tels que $\rho \otimes \sigma_{[a]}$ et $\rho \otimes \sigma_{\left[a_{-}\right]}$sont dans le même espace propre pour $s$ disparaissent. Il ne reste que les contributions des $s$ qui séparent ces 2 représentations. On a alors vu que $\operatorname{Jac}_{(a-1) / 2, \ldots,\left(a_{-}+1\right) / 2} f_{\psi, s}$ sont des éléments de $I_{\text {cusp }}\left(U\left(n-2 d_{\rho}, E / F\right)\right.$ qui proviennent de la distribution stable associée à $\psi_{1}^{\prime} \times \psi_{2}$ dans le groupe endoscopique évident avec l'écriture. Ces éléments sont donc linéairement indépendants et la nullité de (1) assure que pour ces $s$, on a la relation de l'énoncé. Il ne reste donc plus que les termes faisant intervenir une induction $\operatorname{par} \operatorname{St}\left(\rho, a_{-}\right)$avec un élément $f_{\psi_{1}^{\prime} \times \psi_{2}, s^{\prime}}$. Ces termes sont aussi linéairement indépendants et on obtient la relation annoncée dans l'énoncé. 
8.3. Remarques. On reprend les notations $\psi,\left(\rho, a, a_{-}\right)$du paragraphe précédent; en particulier, le fait que $\left(\rho, a, a_{-}\right)$existe entraîne que $\ell(\psi)$ est nécessairement $\geq 2$.

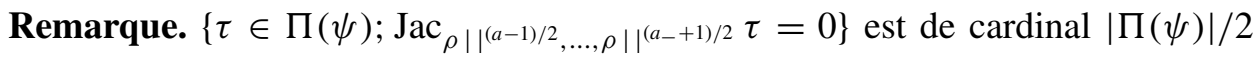
c'est-à-dire $2^{\ell(\psi)-2}$.

Le cardinal de l'ensemble de l'énoncé de la remarque est égal au rang de la matrice rectangulaire $d\left(\tau, s^{\cdot}\right)$, où $\tau$ parcourt cet ensemble et $s \cdot$ parcourt

$$
\left(\operatorname{Cent}_{\mathrm{GL}(n, \mathbb{C})}(\psi) / \mathbb{C}^{*}\right)^{\theta}
$$

où $\mathbb{C}^{*}$ est le centre de $\operatorname{GL}(n, \mathbb{C})$. Et le lemme précédent dit que cette matrice est de rang $1 / 2$ la taille de la matrice carrée $d\left(\tau, s^{*}\right)$ où ici $\tau$ parcourt $\Pi(\psi)$. C'est le résultat annoncé.

\subsection{Liens avec les conjectures d'Arthur.}

8.4.1. Préliminaires. C'est la matrice inverse de la matrice $d\left(\tau, s^{*}\right)$ de 8.1 qui intervient plus spontanément. On note $c\left(s^{*}, \tau\right)$ cette matrice inverse; elle est définie par le fait que pour $s$ un élément $\theta$-invariant du centralisateur de $\psi$, on note $\psi_{s}:=\psi_{1} \times \psi_{2}$ la décomposition de $\psi$ telle que $\psi_{1}$ soit à valeurs dans l'espace propre pour la valeur propre -1 de $s$ et $\psi_{2}$ dans l'espace propre pour la valeur propre +1 . On note $H_{s}$ la donnée endoscopique déterminée par $s$. On considère la représentation $\pi\left(\psi_{s}\right)$ et un prolongement de cette représentation au groupe tordu par $\theta$; cela détermine une distribution stable sur $H_{s}$, notée $\Pi_{H_{s}}^{\text {st }}\left(\psi_{s}\right)$, combinaison linéaire de caractères de séries discrètes de $H_{s}$. Il faut regarder simultanément $\psi_{s}$ et $\psi_{z s}$ où $z$ est la matrice diagonale n'ayant que la valeur propre -1 . Le choix de $\tilde{\pi}\left(\psi_{s}\right)$ induit un choix de $\tilde{\pi}\left(\psi_{z s}\right)$ de telle sorte que $\Pi_{H_{s}}^{\mathrm{st}}\left(\psi_{s}\right)+\Pi_{H_{z s}}^{\mathrm{st}}\left(\psi_{z s}\right)$ soit invariant par le groupe des automorphismes venant de $U(n, E / F)$. On note $\Pi^{s-\text { st }}(\psi)$ l'image de cet élément dans $I_{\text {cusp }}(U(n, E / F))$ et on voit cet élément comme une combinaison linéaire de caractètres de représentations dans $\Pi(\psi)$. Alors, on a par définition :

$$
\Pi^{s-s t}(\psi)=\sum_{\tau \in \Pi(\psi)} c\left(s^{\prime}, \tau\right) \tau .
$$

On reprend la notation $\zeta_{s, \rho, a}$ de 8.2 et on a :

Corollaire. Soit $\tau \in \Pi(\psi)$ et ( $\left.\rho, a, a_{-}\right)$comme en 8.1. Alors,

$$
\operatorname{Jac}_{\rho||^{(a-1) / 2}, \ldots, \rho||^{(a-+1) / 2} \tau \neq 0}
$$

si et seulement si pour tout $s^{*}, c\left(s^{*}, \tau\right)=\zeta_{s, \rho, a} c\left(s^{*} z_{\rho, a}, \tau\right)$.

Par définition pour tout $\tau^{\prime} \in \Pi(\psi)$ et tout $\tau \in \Pi(\psi)$, on a :

$$
\sum_{s} d\left(\tau^{\prime}, s^{\cdot}\right) c\left(s^{*}, \tau\right)=\delta_{\tau^{\prime}, \tau}
$$


où $\delta_{\tau^{\prime}, \tau}$ vaut 0 si $\tau^{\prime} \neq \tau$ et 1 si $\tau^{\prime}=\tau$. Ainsi la condition de l'énoncé est équivalente à ce que (8-2) soit 0 pour tout $\tau^{\prime}$ tel que $\mathrm{Jac}_{\left.\rho\right|^{(a-1) / 2}, \ldots, \rho \|^{\left(a_{-}+1\right) / 2} \tau^{\prime}=0 \text {. Fixons un tel }}$ $\tau^{\prime}$. On regroupe $s^{\prime}$ et $s^{\prime} z_{\rho, a}$ et en appliquant 8.2, la condition (8-1) est équivalente à, pour tout $\tau^{\prime}$ comme ci-dessus :

$$
\sum_{s^{\prime}, s^{\prime} z_{\rho, a}} d\left(\tau^{\prime}, s^{\cdot}\right)\left(c\left(s^{*}, \tau\right)-\zeta_{\rho, a} c\left(s^{\cdot} z_{\rho, a}, \tau\right)\right)=0
$$

Comme la matrice $d\left(\tau^{\prime}, s^{*}\right)$ où $\tau^{\prime}$ est comme ci-dessus et $s^{*}$ parcourt un ensemble de représentants modulo la multiplication par $z_{\rho, a}$ est de rang maximum, on obtient le corollaire.

Pour ne pas compliquer les notations, on note $\left\langle\psi_{s}, \psi_{s}\right\rangle$ la norme elliptique de la représentation virtuelle $\Pi^{s \cdot-\text { st }}(\psi)$, c'est à dire la norme dans $I_{\text {cusp }}(U(n, E / F)$. On a :

Lemme. Pour tout $s, \tau$ comme ci-dessus, $c\left(s^{*}, \tau\right)=d\left(\tau, s^{*}\right)\left\langle\psi_{s}, \psi_{s}\right\rangle$.

Soient $\tau, \tau^{\prime} \in \Pi(\psi)$; on écrit $\left\langle\operatorname{tr} \tau, \operatorname{tr} \tau^{\prime}\right\rangle=\delta_{\tau, \tau^{\prime}}$ où $\langle$,$\rangle est le produit scalaire$ dans $I_{\text {cusp }}(U(n, E / F)$ et cela donne :

$$
\sum_{s \cdot} d\left(\tau, s^{*}\right) d\left(\tau^{\prime}, s^{\cdot}\right)\left\langle\psi_{s}, \psi_{s}\right\rangle=\delta_{\tau, \tau^{\prime}}
$$

Le lemme résulte donc de la définition de $c\left(s^{*}, \tau\right)$ comme inverse de la matrice $d\left(\tau, s^{*}\right)$.

Corollaire. Soit $\tau \in \Pi(\psi)$ et $\left(\rho, a, a_{-}\right)$comme en 8.1. Alors,

$$
\operatorname{Jac}_{\rho||(a-1) / 2}, \ldots, \rho \|^{\left(a_{-}+1\right) / 2} \tau=0
$$

si et seulement si pour tout $s^{*}, c\left(s^{\cdot}, \tau\right)=-\zeta_{s, \rho, a} c\left(s^{*} z_{\rho, a}, \tau\right)\left\langle\psi_{s z_{a}}, \psi_{s z_{a}}\right\rangle\left\langle\psi_{s}, \psi_{s}\right\rangle^{-1}$.

C'est un corollaire immédiat de 8.2.

8.4.2. Normalisation. Jusqu'à présent, nous avons évité de normaliser l'action de $\theta$ sur $\pi(\psi)$, puisque cela n'était pas utile. On pourrait continuer ainsi mais cela complique singulièrement les définitions ; on va donc montrer que la normalisation la plus populaire (à l'aide de modèle de Whittaker) permet de faire disparaître le signe $\zeta_{s, \rho, a}$. On fixe donc un caractère additif de $E^{*}$ et un caractère $\theta$ invariant non dégénéré du groupe unipotent supérieur de $\operatorname{GL}(n, E)$; c'est le caractère usuel puisque $\theta$ respecte un épinglage. Pour faire ceci, il suffit de supposer que la classe de conjugaison de $\psi$ est invariante sous l'action de $\theta$ (vu dualement) et il n'est pas utile de supposer que $\psi$ est $\theta$ discret.

On a étudié les différentes normalisations en [Mœglin et Waldspurger 2006] et il résulte de ces constructions que dans le cas des représentations tempérées tous les choix raisonables donnent le même résultat. C'est donc ce qui se produit ici 
aussi et va permettre de prouver que pour le choix ci-dessus, les signes $\zeta_{s, \rho, a}$ sont tous +1 .

Rappelons une construction simple : soit $\sigma$ une représentation d'un groupe linéaire $\operatorname{GL}\left(n^{\prime}, E\right)$ avec une action de $\theta$, notée $\theta_{\sigma}$. Et soit $\lambda$ une représentation d'un groupe GL $(\ell, E)$; on note $\theta(\lambda)$ l'image de $\lambda \operatorname{par} \theta$. La représentation induite $\lambda \times \sigma \times \theta(\lambda)$ a une action naturelle de $\theta$ provenant de $\theta_{\sigma}$. En effet, on fixe $A_{\lambda}$ un homomorphisme de l'espace où $\lambda$ se réalise dans l'espace où $\theta(\lambda)$ se réalise vérifiant, pour tout $v$ dans l'espace de $\lambda$ et tout $g \in \mathrm{GL}(\ell, F)$

$$
A_{\lambda}(\lambda(\theta(g)) . v)=\theta(\lambda)(g) A_{\lambda}(v) .
$$

Soit $f \in \lambda \times \sigma \times \theta(\lambda)$, c'est-à-dire une fonction sur $\mathrm{GL}\left(n^{\prime}+2 \ell, E\right)$ à valeurs dans l'espace de la représentation $\lambda \otimes \sigma \otimes \theta(\lambda)$. On pose :

$$
\theta(f):=\left(g \in \mathrm{GL}\left(n^{\prime}+2 \ell, E\right) \mapsto\left(A_{\lambda}^{-1} \otimes \theta_{\sigma} \otimes A_{\lambda}\right) \circ \operatorname{inv} f(\theta(g))\right.
$$

où inv est l'application qui envoie $\lambda \otimes \sigma \otimes \theta(\lambda)$ dans $\theta(\lambda) \otimes \sigma \otimes \lambda$ en échangeant les facteurs extrêmes. Cette action de $\theta$ ne dépend que de $\theta_{\sigma}$ et non pas du choix de $A_{\lambda}$.

Soit $\mathscr{E}$ une collection de couples $(\rho, a)$ avec $\rho$ une représentation cuspidale irréductible $\theta$ invariante et $a$ un entier. On fixe $\left(\rho^{\prime}, a^{\prime}\right) \in \mathscr{E}$ avec $a^{\prime} \geq 2$ et on pose $\mathscr{E}^{\prime}$ l'ensemble qui se déduit de $\mathscr{E}$ en remplaçant $\left(\rho^{\prime}, a^{\prime}\right)$ par $\left(\rho^{\prime}, a^{\prime}-2\right)$. On pose :

$$
\pi:=\underset{(\rho, a) \in \mathscr{E}}{\times} \operatorname{St}(\rho, a) ; \pi^{\prime}:=\underset{(\rho, a) \in \in_{\mathscr{E}}^{\prime}}{\times} \operatorname{St}(\rho, a) .
$$

Ces 2 représentations sont munies d'une action de $\theta$ fixant le modèle de Whittaker; on note $\theta_{\pi}^{W}$ et $\theta_{\pi^{\prime}}^{W}$ ces actions. On sait aussi que $\pi$ est un sous-module irréductible de l'induite

$$
\sigma:=\rho^{\prime}||_{E}^{\left(a^{\prime}-1\right) / 2} \times \pi^{\prime} \times \rho^{\prime}||^{-\left(a^{\prime}-1\right) / 2} .
$$

Et $\pi$ est l'unique sous-quotient de cette induite ayant un modèle de Whittaker, ce qui prouve immédiatement le fait que $\pi$ intervient dans cette induite avec multiplicité 1 . Comme on a supposé que $\rho^{\prime}$ est $\theta$ invariante, l'induite (8-3) a une action de $\theta$ qui provient de $\theta_{\pi^{\prime}}^{W}$. On note $\theta$ cette action.

Lemme. L'action $\theta_{\pi}^{W}$ est la restriction de $\theta$ à $\pi$ pour l'inclusion de $\pi$ dans (8-3).

On note $V^{\prime}$ l'espace de $\rho^{\prime}||_{E}^{\left(a^{\prime}-1\right) / 2} \otimes \pi^{\prime} \otimes \rho^{\prime}||^{-\left(a^{\prime}-1\right) / 2}$ et $\ell_{V^{\prime}}^{W}$ une fonctionnelle de Whittaker sur $V^{\prime}$; cela étend la fonctionnelle de Whittaker sur $\pi^{\prime}$ de façon invariante sous inv utilisé dans les constructions ci-dessus. On construit une fonctionnelle de Whittaker sur $V$, l'espace de $\sigma$, de façon standard (on rappelera sa construction) $\ell_{V}^{W}$. On note $n$ l'entier tel que $\pi$ soit une représentation de $\operatorname{GL}(n, E)$ et $N$ le groupe des matrices unipotentes supérieures de $\operatorname{GL}(n, E)$; on note $\chi$ le caractère de $N, \theta$-invariant qui sert à définir le modèle de Whittaker. D'abord 
$\theta_{\pi}^{W}$ est l'action de $\theta$ qui induit l'identité sur l'espace vectoriel de dimension 1, $V / N_{\chi} V$ (module de Jacquet tordu). Pour construire $\ell_{V}^{W}$ on identifie $V$ à un espace de fonction de GL $(n, E)$ à valeurs dans $V^{\prime}$, covariante pour l'action du sousgroupe parabolique supérieur de Levi isomophe à $M:=\operatorname{GL}\left(d_{\lambda}, E\right) \times \operatorname{GL}\left(n^{\prime}, E\right) \times$ $\mathrm{GL}\left(d_{\lambda}, E\right)$ (avec des notations évidentes). On note $w$ l'élément du groupe de Weyl de $\operatorname{GL}(n, E)$ de longueur minimale dans sa double classe modulo le groupe de Weyl de $M$ qui rend négatif toutes les racines simples hors de $M$.

On note $N_{P}$ le radical unipotent du parabolique qui sert à l'induction. Et on pose pour tout $f \in V$ :

$$
\ell_{V}^{W}(f):=\int_{N_{P}} \ell_{V^{\prime}}^{W} f(w n) \chi(n) d n .
$$

L'intégrale se fait sur un compact et est donc une somme finie qui se factorise par $V / N_{\chi} V$. Pour vérifier que l'action de $\theta$ sur (8-3) prolongeant canoniquement $\theta_{\pi^{\prime}}^{W}$ induit aussi l'action triviale sur $V / N_{\chi} V$, il suffit de calculer explicitement $\ell_{V}^{W}(\theta . f)$; le résultat est la même intégrale mais avec $w$ remplacé par $\theta(w)$. On vérifie que $\theta(w)=z w$ avec $z$ un élément du centre de $M$ de la forme $z_{1} \times 1 \times z_{1}$ avec $z_{1}$ valant $\pm \operatorname{Id}_{d_{\lambda}}$. La représentation induisante a un caractère central trivial sur $z$ d'où l'invariance

$$
\ell_{V}^{W}(f)=\ell_{V}^{W}(\theta(f))
$$

Cela assure que $\theta(f)$ et $f$ ont même image dans $V / N_{\chi} V$ comme cherché.

Corollaire. Toutes les actions de $\theta$ étant fixées par la normalisation de Whittaker, les signes $\zeta_{s, \rho, a}$ de 8.2 sont tous égaux à +1 .

Il faut d'abord se ramener au cas où $s=1$; cela résulte du fait que le transfert commute à la prise du module de Jacquet; voir 1.6. Pour $s=1$, avec $(\rho, a)$ comme dans l'énoncé on vient de montrer que l'inclusion :

$\underset{\left(\rho^{\prime}, a^{\prime}\right) \in \mathscr{E}}{\times} \operatorname{St}(\rho, a) \hookrightarrow \rho\left\|_{E}^{(a-1) / 2} \times\left(\underset{\substack{\left(\rho^{\prime}, a^{\prime}\right) \in \\ \mathscr{E}-\{(\rho, a)\}}}{\times} \operatorname{St}\left(\rho^{\prime}, a^{\prime}\right) \times \operatorname{St}(\rho, a-2)\right) \times \rho\right\|_{E}^{-(a-1) / 2}$

est compatible aux actions de $\theta$ quand sur le membre de droite on met l'action prolongeant canoniquement l'action de $\theta$ sur la représentation entre parenthèse. Ce prolongement canonique est compatible au module de Jacquet, par définition puisque

$$
\operatorname{Jac}_{\rho \mid I_{E}^{(a-1) / 2}}^{\theta}(2)=\underset{\substack{\left(\rho^{\prime}, a^{\prime}\right) \in \\ \mathscr{E}-\{(\rho, a)\}}}{\times} \operatorname{St}\left(\rho^{\prime}, a^{\prime}\right) \times \operatorname{St}(\rho, a-2)
$$

s'obtient en évaluant à l'origine. Si $a_{-}$(notation de 8.2) vaut $a-2$, on a immédiatement $\zeta_{1, \rho, a}=+1$ et sinon on obtient le corollaire en réitérant cette construction. 
8.4.3. Caractère de $A(\psi)$. On fixe $\psi$ un morphisme $\theta$-discret et stable de $W_{E} \times$ $\operatorname{SL}(2, \mathbb{C})$ dans $\operatorname{GL}(n, \mathbb{C})$. D'où un paquet de représentations $\Pi(\psi)$ de $U(n, E / F)$. On a introduit $A(\psi)$ un sous-groupe du centralisateur de $\psi$ en 8.4.1. Pour comprendre ce qui se passe, on va tout de suite construire un sous-groupe $S_{0}$ du centralisateur de $\psi$ de telle sorte que :

$$
S_{0} \cap A(\psi)=\{1\}, \quad\left(\operatorname{Cent}_{\mathrm{GL}(n, \mathbb{C})} \psi\right)^{\theta}=S_{0} A(\psi) .
$$

On écrit $\psi$ comme une somme de représentations irréductibles $\rho \otimes \sigma_{[a]}$ de $W_{E} \times$ $\operatorname{SL}(2, \mathbb{C})$ et on note $\mathscr{E}$ l'ensemble des couples $(\rho, a)$ intervenant. On sait que le centralisateur de $\psi$ dans $\operatorname{GL}(n, \mathbb{C})$ est le produit des groupes $\mathbb{C}^{*}$ agissant scalairement sur chaque espace des sous-représentations $\rho \otimes \sigma_{[a]}$; quand on considère les éléments $\theta$-invariants de ce groupe, il reste \pm 1 . On considère $\mathscr{E}_{\text {imp }}$ l'ensemble des couples $(\rho, a) \in \mathscr{E}$ tel que $a$ soit impair. On rappelle que cette condition est équivalente à ce que $L\left(\rho, r_{A}^{\prime}, s\right)$ n'ait pas de pôle en $s=0$. On remarque que $A(\psi)$ contient tous les facteurs \pm 1 relatifs aux éléments de $\mathscr{E}-\mathscr{E}_{\text {imp. }}$. Donc en particulier si $\mathscr{E}_{\mathrm{imp}}=\varnothing,(8-4)$ est réalisé avec $S_{0}=\varnothing$ et $Z$ est alors inclus dans $A(\psi)$. Supposons donc que $\mathscr{E}_{\mathrm{imp}} \neq \varnothing$. On fixe $\rho$ et un nombre impair d'entiers $A_{\rho}$ tel que $(\rho, a) \in \mathscr{E}_{\text {imp }}$ pour tout $a \in A_{\rho}$. On note $z_{\rho}$ l'élément du centralisateur de $\psi$ produit des éléments -1 relatifs à ces représentations $\rho \otimes \sigma_{[a]}$ pour $a$ parcourant $A_{\rho}$. On note alors $S_{0}$ le groupe engendré par ces éléments $z_{\rho}$. Evidemment la définition n'est pas du tout canonique puisqu'elle dépend du choix de $A_{\rho}$ mais (8-4) est clair. Un choix qui en vaut bien un autre est de prendre $A_{\rho}$ réduit à un élément et précisément à l'élément le plus petit possible.

Théorème. Pour tout $\tau \in \Pi(\psi)$, il existe un unique caractère, $\varepsilon_{\tau}$ de $A(\psi)$ tel que pour tout $s \in\left(\operatorname{Cent}_{\mathrm{GL}(n, \mathbb{C})} \psi\right)^{\theta}$ et tout $a \in A(\psi)$, il existe un réel strictement positif $\alpha$ avec l'égalité :

$$
c\left(s^{\cdot} a, \tau\right)=\varepsilon_{\tau}(a) c\left(s^{*}, \tau\right) \alpha .
$$

Montrons d'abord l'unicité de $\tau$ : on fixe $s=1$, on sait alors que $c(1 \cdot \tau) \neq 0$ et $\varepsilon_{\tau}(a)$ est alors le signe de $c(a, \tau) / c(1 \cdot \tau)$ qui est calculé en fonction des modules de Jacquet de $\tau$ grâce aux 2 propriétés démontrée en 8.4.1. On peut donc remplacer 1 dans cette définition par n'importe quel élément de $A(\psi)$. Remarquons maintenant que cette application signe définit donc bien un caractère. En appliquant ces mêmes références, où on peut prendre $s$ quelconque, on obtient le théorème. On conjecture que $c\left(s^{*}, \tau\right)= \pm 1$ et que $\alpha=1$ mais ce n'est pas prouvé ici. Toutefois, on peut calculer $\alpha$ comme quotient de normes (que l'on ne sait pas calculer).

8.4.4. Classification des représentations cuspidales. On reprend les notations $\psi$, $A(\psi)$ et $\varepsilon_{\tau}$ de 8.4.3. On note $\varepsilon_{\text {alt }}$ l'unique caractère de $A(\psi)$ qui vaut -1 sur tout générateur $z_{\rho, a}$ défini en 8.2. On a défini le fait que $\psi$ soit sans trou dans l'introduction; on a vu que si $\tau \in \Pi(\psi)$ le support cuspidal étendu de $\tau$ se calcule 
avec la décomposition en représentations irréductibles de $\psi$. On a donné la forme du support cuspidal étendu d'une représentation cuspidale en et il en résulte que les seuls morphismes $\psi$ tels que $\Pi(\psi)$ contienne une représentation cuspidale sont les $\psi$ qui sont sans trou.

Théorème. On suppose que $\psi$ est sans trou. La représentation $\tau$ est cuspidale si et seulement si $\varepsilon_{\tau}=\varepsilon_{\text {alt }}$. Le nombre de représentations cuspidales dans $\Pi(\psi)$ est le cardinal de l'ensemble des caractères de $\left(\operatorname{Cent}_{\mathrm{GL}(n, \mathbb{C})} \psi\right)^{\theta} /\{\mathrm{Id},-\mathrm{Id}\}$ dont la restriction à $A(\psi)$ vaut $\varepsilon_{\text {alt }}$.

La première partie du théorème résulte de 5.2 montrant que $\varepsilon_{\tau}=\varepsilon_{\text {alt }}$ si et seulement si tous les modules de Jacquet de $\tau$ sont nuls. Montrons la deuxième partie : le premier point à remarquer est que si $Z=\{\mathrm{Id},-\mathrm{Id}\}$ est inclus dans $A(\psi)$, la restriction de $\varepsilon_{\tau}$ à $Z$ est nécessairement l'identité. Il y a deux cas à distinguer, le premier cas est celui où $Z$ est inclus dans $A(\psi)$ et où $\varepsilon_{\text {alt }}$ n'est pas de restriction triviale à $Z$. Dans ce cas, $\Pi(\psi)$ ne contient pas de représentations cuspidales et la fin du théorème est claire. Dans le cas opposé, on note encore $\varepsilon_{\text {alt }}$ le caractère du groupe $Z A(\psi)$ qui vaut $\varepsilon_{\text {alt }}$ sur $A(\psi)$ et est trivial sur $Z$. On fixe $S_{0}^{\prime}$ un sousensemble de $S_{0}$ tel que $S_{0} A(\psi)=S_{0}^{\prime} A(\psi) Z$. Pour tout $\tau \in \Pi(\psi)$ on note encore $\varepsilon_{\tau}$ le caractère de $Z A(\psi)$ dont la restriction à $A(\psi)$ est $\varepsilon_{\tau}$ et qui est trivial sur $Z$. On considère l'application qui à $\tau \in \Pi(\psi)$ associe l'élément $\left(c\left(s_{0}^{\prime}, \tau\right) \in \mathbb{C}^{\left|S_{0}^{\prime}\right|}\right), \varepsilon_{\tau}$ c'est à dire un élément de $\mathbb{C}^{\left|S_{0}^{\prime}\right|}$ et un caractère de $Z A(\psi)$ trivial sur $Z$. Le fait que la matrice est de rang $c\left(s^{*}, \tau\right)$ et inversible se traduit par le fait que pour tout caractère $\varepsilon_{0}$ de $Z A(\psi)$ trivial sur $Z$, la matrice $\left\{c\left(s_{0}, \tau\right) ; s_{0} \in S_{0}^{\prime}, \varepsilon_{\tau}=\varepsilon_{0}\right\}$ est de rang $\left|S_{0}^{\prime}\right|$. Ceci est a fortiori vrai pour $\varepsilon_{0}=\varepsilon_{\text {alt }}$ et donne le résultat cherché.

\section{Références}

[Arthur 1993] J. Arthur, "On elliptic tempered characters", Acta Math. 171 :1 (1993), 73-138. MR 94i :22038 Zbl 0822.22011

[Arthur 1996] J. Arthur, “On local character relations”, Selecta Math. (N.S.) 2 :4 (1996), 501-579. MR 2000a :22017 Zbl 0923.11081

[Arthur 2005] J. Arthur, "An introduction to the trace formula", pp. 1-263 dans Harmonic analysis, the trace formula, and Shimura varieties, édité par D. E. James Arthur et R. Kottwitz, Clay Math. Proc. 4, Amer. Math. Soc., Providence, RI, 2005. MR 2007d :11058

[Clozel 1982] L. Clozel, "Changement de base pour les représentations tempérées des groupes réductifs réels”, Ann. Sci. École Norm. Sup. (4) 15 :1 (1982), 45-115. MR 84j :22015 Zbl 0516.22010

[Goldberg 1994] D. Goldberg, "Some results on reducibility for unitary groups and local Asai $L$ functions", J. Reine Angew. Math. 448 (1994), 65-95. MR 95g :22031 Zbl 0815.11029

[Harris et Labesse 2004] M. Harris et J.-P. Labesse, "Conditional base change for unitary groups", Asian J. Math. 8 :4 (2004), 653-683. MR 2006g:11098 Zbl 1071.22025

[Kottwitz et Shelstad 1999] R. E. Kottwitz et D. Shelstad, Foundations of twisted endoscopy, Astérisque 255, Soc. math. de France, Paris, 1999. MR 2000k :22024 Zbl 0958.22013 
[Labesse 2004] J.-P. Labesse, "Stable twisted trace formula : elliptic terms", J. Inst. Math. Jussieu 3 :4 (2004), 473-530. MR 2005g :11084 Zbl 1061.11025

[Laumon et Ngo 2004] G. Laumon et B.-C. Ngo, "Le lemme fondamental pour les groupes unitaires", prépublication, 2004.

[Mezo 2004] P. Mezo, “Twisted trace Paley-Wiener theorems for special and general linear groups", Compos. Math. 140 :1 (2004), 205-227. MR 2004e :22010 Zbl 1050.22013

[Mœglin 2002] C. Mœglin, "Sur la classification des séries discrètes des groupes classiques $p$ adiques : paramètres de Langlands et exhaustivité", J. Eur. Math. Soc. 4 :2 (2002), 143-200. MR 2003g :22021 Zbl 1002.22009

[Mœglin 2003] C. Mœglin, "Points de réductibilité pour les induites de cuspidales", J. Algebra 268 :1 (2003), 81-117. MR 2005a :22012 Zbl 1028.22016

[Mœglin et Tadić 2002] C. Mœglin et M. Tadić, "Construction of discrete series for classical $p$-adic groups”, J. Amer. Math. Soc. 15 :3 (2002), 715-786. MR 2003g :22020 Zbl 0992.22015

[Mœglin et Waldspurger 2006] C. Mœglin et J.-L. Waldspurger, "Sur le transfert des traces d'un groupe classique $p$-adique à un groupe linéaire tordu", Selecta Math. (N.S.) 12 :3-4 (2006), 433515. MR 2305609 Zbl 05189557

[Schneider et Stuhler 1997] P. Schneider et U. Stuhler, "Representation theory and sheaves on the Bruhat-Tits building”, Inst. Hautes Études Sci. Publ. Math. 85 (1997), 97-191. MR 98m :22023 Zbl 0892.22012

[Shahidi 1990] F. Shahidi, “A proof of Langlands' conjecture on Plancherel measures ; complementary series for p-adic groups", Ann. of Math. (2) 132 :2 (1990), 273-330. MR 91m :11095 Zbl 0780.22005

[Silberger 1980] A. J. Silberger, "Special representations of reductive $p$-adic groups are not integrable”, Ann. of Math. (2) 111 :3 (1980), 571-587. MR 82k :22015 Zbl 0437.22015

[Waldspurger 1997] J.-L. Waldspurger, "Le lemme fondamental implique le transfert", Compositio Math. 105 :2 (1997), 153-236. MR 98h :22023 Zbl 0871.22005

[Waldspurger 2006a] J.-L. Waldspurger, "Endoscopie et changement de caractéristique", J. Inst. Math. Jussieu 5 :3 (2006), 423-525. MR 2007h :22007 Zbl 1102.22010

[Waldspurger 2006b] J.-L. Waldspurger, "L'endoscopie tordue n'est pas si tordue", prépublication, 2006. À paraître dans Mem. Amer. Math. Soc.

[Waldspurger 2007] J.-L. Waldspurger, "Le groupe $\mathbf{G L}_{N}$ tordu, sur un corps $p$-adique, I", Duke Math. J. 137 :2 (2007), 185-234. MR MR2309147 Zbl 1113.22013

Received January 8, 2007.

\section{Colette Meglin}

30 RUE DE Plaisance

F-94000 CRETEIL

FRANCE

moeglin@math.jussieu.fr

http://www.math.jussieu.fr/ moeglin 